Supplement of Atmos. Chem. Phys. Discuss., 14, 17101-17159, 2014

http://www.atmos-chem-phys-discuss.net/14/17101/2014/

doi:10.5194/acpd-14-17101-2014-supplement

(c) Author(s) 2014. CC Attribution 3.0 License.

(c) (1)

Supplement of

\title{
Chemical climatology of the southeastern United States, 1999-2013
}

\author{
G. M. Hidy et al.
}

Correspondence to: G. M. Hidy (dhidy113@comcast.net) 


\section{Chemical Climatology of the Southeastern United States, 1999-2013}

\section{Supplemental Information}

\section{Overview}

The following material supplements the manuscript, "Chemical Climatology of the Southeastern United States, 1999-2013." The tables and figures provide supporting detail to the discussion in this paper that can be used by readers in further interpreting the observations in the SEARCH region and at the CTR site.

The first part includes four tables covering (1) the basic instrumentation of the SEARCH sites, including CTR, (2) a table of a measure of apparent sulfate neutralization as ammonium salts, (3) a table comparing rural and urban aerometric observations, and (4) a survey of rural NMOC from the literature in the southeastern region.

The series of figures include: (1) a photograph of the CTR site, (2) annual emission trends of various gases and particle species, (3) a normalized seasonal plot for particle mass and composition, (4) an empirical relationship between $\mathrm{O}_{3}$ and $\mathrm{OH}$, (5) trends in $\mathrm{PM}_{2.5}$ composition for the SEARCH network and for CTR, (6) EC and OC concentrations at CTR compared with emissions, (7) mean EC and OC concentration trends at certain SEARCH sites, (8) trends in annual fires in different states, (9) time series for optical properties of aerosols at CTR, (10) trends in annual contributions to extinction coefficient for SEARCH, and for June-July period, (11) diurnal variations in $\mathrm{SO}_{2}$ and $\mathrm{NO}_{\mathrm{x}}$ emissions from statewide electric power generation, (12) diurnal variations of motor vehicle emissions, (13) trend-distributions of meteorological parameters for CTR, (14) trenddistributions of gaseous constituents for CTR, (15) trend-distributions of particulate components for CTR, (16) composition reactive nitrogen balance for CTR, (17) diurnal difference between dry and wet light scattering as "water" content, (18) mean diurnal concentrations of selected NMOC groups, (19) June average isoprene and $\alpha$-pinene concentrations in rural Alabama. 
Table S1. Basic measurements at CTR and other SEARCH sites from 1999-2013. Sites having instrument differences are noted under "Comments." (PNS, OAK, and GFP were decommissioned at the end of 2009, 2010, and 2012, respectively).

a. Continuous meteorology at SEARCH sites (5 and 60 minute reporting intervals).

\begin{tabular}{|c|c|c|c|c|}
\hline Parameter & Method & Instrument & $\begin{array}{c}\text { Sampling } \\
\text { Height } \\
\text { (magl) }\end{array}$ & Comments \\
\hline \multirow[t]{2}{*}{ Temperature } & & Vaisala HMT3303 & 2 & $2 \mathrm{~m}$ added in 2011 \\
\hline & platinum RTD & Paroscientific Met 4B & 9 & \\
\hline \multirow[t]{2}{*}{ Relative humidity } & & Vaisala HMT3303 & 2 & $2 \mathrm{~m}$ added in 2011 \\
\hline & thin film capacitance & Paroscientific Met 4B & 9 & \\
\hline Barometric pressure & $\begin{array}{l}\text { quartz crystal } \\
\text { resonator }\end{array}$ & Paroscientific Met 4B & 9 & \\
\hline Surface wetness & conductivity & Decagon LSW-1 & 2 & Added in 2011 \\
\hline \multirow[t]{2}{*}{ Wind Speed/Direction } & & & & \\
\hline & sonic anemometer & RMYoung 8100 & 10 & \\
\hline Solar Radiation (PAR) & pyranometer & Licor-200X & 2 & \\
\hline Precipitation & gravimetry & ETI NOAH-IV & 2 & \\
\hline Aerosol/cloud & & & $50-$ & GFP and OLF 2012-13 \\
\hline layers/mixing depth & laser ceilometer & JenOptik CHM 15k & 15,000 & CTR and BHM 2013-14 \\
\hline
\end{tabular}

b. Continuous trace gases at SEARCH sites (5 and 60 minute reporting intervals).

\begin{tabular}{|c|c|c|c|c|}
\hline Parameter & Method & Instrument & $\begin{array}{c}\text { Sampling } \\
\text { Hgt. } \\
\text { (magl) }\end{array}$ & Comments \\
\hline $\mathrm{O}_{3}$ & UV absorption & Thermo-Scientific 49i & 10 & \\
\hline $\mathrm{CO}$ & NDIR & Thermo-Scientific 48i & 10 & \\
\hline $\mathrm{SO}_{2}$ & Pulsed UV fluorescence & Thermo-Scientific $43 \mathrm{i}$ & 10 & \\
\hline $\mathrm{NO}, \mathrm{NOy}$ & Mo reduction/CL & Thermo-Scientific $42 \mathrm{i}$ & 10 & \\
\hline $\mathrm{NO}_{2}$ & Blue LED photolysis/CL & Thermo-Scientific $42 \mathrm{i}$ & 10 & \\
\hline $\mathrm{HNO}_{3}$ & $\begin{array}{l}\text { Denuder difference/Mo } \\
\text { reduction/CL }\end{array}$ & Thermo-Scientific $42 \mathrm{i}$ & 10 & \\
\hline PANS/Alkylnitrates & $\begin{array}{l}\text { Thermal decomposition/Blue } \\
\text { LED photolysis/CL }\end{array}$ & Thermo-Scientific $42 \mathrm{i}$ & 5 & $\begin{array}{l}\text { CTR only, } \\
\text { starting } 6 / 13\end{array}$ \\
\hline $\mathrm{NH}_{3}$ & $\begin{array}{l}\text { Denuder difference/Pt } \\
\text { oxidation/Mo reduction/CL }\end{array}$ & Thermo-Scientific $42 \mathrm{i}$ & 5 & Added in 2007 \\
\hline
\end{tabular}

$\mathrm{CL}=\mathrm{NO}-\mathrm{O}_{3}$ chemiluminescence 
c. Continuous PM at SEARCH sites (5 and 60 minute reporting intervals).

\begin{tabular}{|c|c|c|c|c|}
\hline Parameter & Method & Instrument & $\begin{array}{c}\text { Sampling } \\
\text { Hgt. (magl) }\end{array}$ & Comments \\
\hline $\mathrm{PM}_{2.5}$ mass (dry) & Oscillating micro-balance & R\&P $1400 \mathrm{a} / \mathrm{b}$ TEOM & 5 & \\
\hline $\mathrm{PM}_{10}$ mass (dry) & Oscillating micro-balance & $\mathrm{R} \& \mathrm{P} 1400 \mathrm{a} / \mathrm{b}$ TEOM & 5 & BHM/JST only \\
\hline $\mathrm{PM}_{2.5} \mathrm{SO}_{4}^{2-}$ & $\begin{array}{l}\text { Reduction to } \mathrm{SO}_{2} \text { on Inconel/UV } \\
\text { fluorescence }\end{array}$ & & 5 & Added in $2002-3$ \\
\hline $\mathrm{PM}_{2.5} \mathrm{NO}_{3}^{-}$ & $\begin{array}{l}\text { Filter difference/Mo } \\
\text { reduction/CL }\end{array}$ & & 5 & Added in $2002-3$ \\
\hline $\mathrm{PM}_{2.5} \mathrm{NH}_{4}^{+}$ & $\begin{array}{l}\text { Filter difference/Pt oxidation/Mo } \\
\text { reduction/CL }\end{array}$ & & 5 & Added in $2002-3$ \\
\hline $\mathrm{PM}_{2.5} \mathrm{TC} / \mathrm{EC}$ & Oxidation to CO2/NDIR (TC) & Sunset Particulate & 5 & 60-min only \\
\hline $\mathrm{B}_{\text {scat }}(\mathrm{dry})$ & Nephelometry @530nm & $\begin{array}{l}\text { Radiance Research } \\
\text { M903 }\end{array}$ & 5 & Added in 2000 \\
\hline $\mathrm{B}_{\text {scat }}$ (ambient) & Nephelometry@530 nm & Optec NGN2a & 5 & Added in 2013 \\
\hline $\mathrm{B}_{\mathrm{abs}}$ & $\begin{array}{l}\text { Optical absorption @ } 880 \text { nm } \\
\text { and } 370 \text { nm (quartz filter) }\end{array}$ & $\begin{array}{l}\text { Magee Sci. AE2x } \\
\text { aethalometer }\end{array}$ & 5 & Added in 2000 \\
\hline
\end{tabular}

d. Discrete particle and gas measurements at SEARCH sites (24-hour sampling intervals; daily or 1-in-3 sample schedule; sample height 5 magl).

\begin{tabular}{|c|c|c|c|c|}
\hline Parameter & Sample Medium & Sample Device & Analytical Method & Comments \\
\hline $\mathrm{PM}_{2.5}$ mass & Teflon filter & $\begin{array}{l}\text { Thermo R\&P } 2025 \\
\text { FRM }\end{array}$ & $\begin{array}{l}\text { Gravimetry } \\
\text { (w/buoyancy corr.) }\end{array}$ & \\
\hline $\mathrm{PM}_{2.5}$ anions/cations & Denuded Teflon filter & ARA PCM1 & Ion chromatography & 3 anions, 5 cations \\
\hline $\mathrm{PM}_{2.5}$ volatile anions & Backup nylon filter & ARA PCM1 & Ion chromatography & $\mathrm{Cl}^{-}$and $\mathrm{NO}_{3}^{-}$ \\
\hline $\mathrm{PM}_{2.5}$ trace elements & Teflon filter & $\begin{array}{l}\text { Thermo R\&P } 2025 \\
\text { FRM }\end{array}$ & $\mathrm{X}$-ray fluorescence & 10 elements \\
\hline $\begin{array}{l}\mathrm{PM}_{2.5} \text { water-sol. } \\
\text { trace elements }\end{array}$ & Denuded Teflon Filter & ARA PCM1 & ICP-MS & 12 elements \\
\hline $\mathrm{PM}_{2.5} \mathrm{EC} / \mathrm{OC}$ & Denuded quartz filter & ARA PCM3 & $\begin{array}{l}\text { Thermo-optical } \\
\text { reflectance }\end{array}$ & 7 fractions \\
\hline $\begin{array}{l}\text { PM2.5 Speciated OC } \\
\sim 125 \text { cpds. }\end{array}$ & Quartz filter & DRI Medvol & $\begin{array}{l}\text { Thermal } \\
\text { desorption/GC-MS }\end{array}$ & $\begin{array}{l}\text { BHM and JST } \\
2006-10\end{array}$ \\
\hline $\mathrm{PM}_{\text {coarse }}$ mass & Teflon Filter & $\begin{array}{l}\text { Andersen Inst. SA- } \\
241 \text { dichotomous } \\
\text { sampler }\end{array}$ & $\begin{array}{l}\text { Gravimetry } \\
\text { (w/buoyancy corr.) }\end{array}$ & \\
\hline $\begin{array}{l}\mathrm{PM}_{\text {coarse }} \\
\text { anions/cations }\end{array}$ & “ & “ & Ion chromatography & \\
\hline $\begin{array}{l}\mathrm{PM}_{\text {coarse }} \text { trace } \\
\text { elements }\end{array}$ & “ & “ & X-ray fluorescence & \\
\hline $\begin{array}{l}\mathrm{PM}_{\text {coarse }} \text { water-sol. } \\
\text { trace elements }\end{array}$ & “ & “ & ICP-MS & \\
\hline $\mathrm{PM}_{\text {coarse }} \mathrm{EC} / \mathrm{OC}$ & Quartz filter & “ & $\begin{array}{l}\text { Thermo-optical } \\
\text { reflectance }\end{array}$ & $\begin{array}{l}\text { Limited sites, } \\
\text { time periods }\end{array}$ \\
\hline $\mathrm{NH}_{3}$ & $\begin{array}{l}\text { Citric acid annular } \\
\text { denuder }\end{array}$ & $\begin{array}{l}\text { Shop-built pump/flow } \\
\text { controller }\end{array}$ & Ion chromatography & Added in 2004 \\
\hline NMOC/OVOC & Summa canister & “ & GC-MS & $\begin{array}{l}\text { JST 1998-08 } \\
\text { BHM 2006-10 }\end{array}$ \\
\hline
\end{tabular}


Table S2. Mean ratios of $\mathrm{SO}_{4}$-S:XRF-S ( $\mathrm{SO}_{4} / 3^{*} \mathrm{XRF}$ S) computed from $1999-2010$ SEARCH data. The standard errors (SE) of the monthly means ranged from 0.005 to 0.03 (mean 0.01). Linear regression forced through the origin yielded slope values ranging from $0.931 \pm 0.006$ (December) to $1.043 \pm 0.004$ (August) ( $n=14,237$ samples). Note that the filter data used for comparison derive from two different SEARCH samplers (FRM and PCM1). Since the PCM1 sampler utilizes an $\mathrm{SO}_{2}$ denuder and the FRM sampler does not, the comparison has a sampling ambiguity that is not accounted for in the ratios.

\begin{tabular}{cccccccccc}
\hline Month & BHM & CTR & GFP & JST & OAK & OLF & PNS & YRK & Average \\
\hline Jan & 0.98 & 0.93 & 0.96 & 0.94 & 0.93 & 0.93 & 0.92 & 0.96 & 0.94 \\
Feb & 0.97 & 0.95 & 0.98 & 0.96 & 0.97 & 0.94 & 0.97 & 0.98 & 0.96 \\
Mar & 0.99 & 0.98 & 0.96 & 0.99 & 0.98 & 0.97 & 0.97 & 1.00 & 0.98 \\
Apr & 1.02 & 0.98 & 0.98 & 1.05 & 1.00 & 0.99 & 0.99 & 1.01 & 1.00 \\
May & 1.02 & 0.99 & 1.00 & 1.02 & 1.00 & 1.03 & 0.99 & 1.04 & 1.01 \\
June & 1.06 & 1.00 & 1.01 & 1.03 & 1.02 & 1.00 & 1.00 & 1.02 & 1.02 \\
July & 1.04 & 0.98 & 1.05 & 1.04 & 1.01 & 1.00 & 0.99 & 1.03 & 1.02 \\
Aug & 1.05 & 1.01 & 1.01 & 1.04 & 1.03 & 0.99 & 1.02 & 1.04 & 1.02 \\
Sep & 1.03 & 1.01 & 0.97 & 1.03 & 1.03 & 1.02 & 0.99 & 1.01 & 1.01 \\
Oct & 1.01 & 0.98 & 0.98 & 1.03 & 0.98 & 0.96 & 0.96 & 1.00 & 0.99 \\
Nov & 0.97 & 0.95 & 0.97 & 0.99 & 0.99 & 0.96 & 0.94 & 1.00 & 0.97 \\
Dec & 1.04 & 0.94 & 0.94 & 0.98 & 0.94 & 0.92 & 0.93 & 0.97 & 0.96 \\
\hline
\end{tabular}


Table S3. Seasonal mean concentrations for a period (2004 - 2007) when all sites were operational and reported measurements of each of the tabled species: $\mathrm{SO}_{2}$ and $\mathrm{SO}_{4}$ as $\mathrm{SO}_{2} ; \mathrm{NH}_{3}$ and $\mathrm{NH}_{4}$ as $\mathrm{NH}_{3} ; \mathrm{NO}, \mathrm{NO}_{2}, \mathrm{HNO}_{3}, \mathrm{NO}_{3}$, and $\mathrm{NO}_{\mathrm{y}}$ species as $\mathrm{NO}_{2}$. Units are $\mu \mathrm{g}$ $\mathrm{m}^{-3}$. Coastal sites are above the heavy line. For each site pair, the non-urban site is listed first. Source: Blanchard et al. (2013a).

\begin{tabular}{|c|c|c|c|c|c|c|c|c|c|c|c|c|}
\hline Site & Season $^{\mathrm{a}}$ & $\mathrm{SO}_{2}$ & $\mathrm{SO}_{4}$ & $\mathrm{NH}_{3}$ & $\mathrm{NH}_{4}$ & $\mathrm{NO}$ & $\mathrm{NO}_{2}$ & $\mathrm{NO}_{\mathrm{V}}$ & $\mathrm{HNO}_{3}$ & $\mathrm{pNO}_{3}$ & $\mathrm{EC}$ & $\mathrm{OM}$ \\
\hline \multirow[t]{4}{*}{ OAK } & Spring & 3.5 & 2.3 & 0.4 & 1.0 & 0.2 & 2.6 & 5.2 & 0.7 & 0.2 & 0.5 & 3.5 \\
\hline & Summer & 2.8 & 3.0 & 0.2 & 1.1 & 0.2 & 2.1 & 4.1 & 0.4 & 0.1 & 0.4 & 4.0 \\
\hline & Autumn & 4.4 & 2.4 & 0.2 & 1.0 & 0.2 & 3.0 & 5.4 & 0.5 & 0.2 & 0.5 & 3.7 \\
\hline & Winter & 5.2 & 1.5 & 0.2 & 0.8 & 0.4 & 5.3 & 8.2 & 0.7 & 0.4 & 0.8 & 4.7 \\
\hline \multirow[t]{4}{*}{ GFP } & Spring & 3.0 & 2.3 & 0.7 & 1.1 & 2.3 & 11.1 & 16.1 & 0.2 & 0.3 & 0.6 & 2.9 \\
\hline & Summer & 3.9 & 2.8 & 0.6 & 1.1 & 1.7 & 12.3 & 16.7 & 0.6 & 0.2 & 0.6 & 3.4 \\
\hline & Autumn & 6.1 & 2.5 & 0.6 & 1.2 & 4.4 & 15.8 & 23.7 & 0.5 & 0.3 & 0.8 & 3.7 \\
\hline & Winter & 5.9 & 1.4 & 0.5 & 0.8 & 8.7 & 16.3 & 29.1 & 0.3 & 0.5 & 0.7 & 3.1 \\
\hline \multirow[t]{4}{*}{ OLF } & Spring & 4.2 & 2.3 & 0.5 & 1.1 & 1.0 & 8.1 & 11.3 & 0.5 & 0.3 & 6 & 3.3 \\
\hline & Summer & 4.5 & 2.9 & 0.4 & 1.2 & 2.3 & 8.8 & 13.0 & 0.5 & 0.2 & 0.7 & 4.1 \\
\hline & Autumn & 7.9 & 2.5 & 0.3 & 1.1 & 1.5 & 11.3 & 14.9 & 0.4 & 0.2 & 0.8 & 4.1 \\
\hline & Winter & 7.0 & 1.4 & 0.3 & 0.7 & 1.8 & 11.8 & 15.5 & 0.2 & 0.4 & 0.6 & 3.1 \\
\hline \multirow[t]{4}{*}{ PNS } & Spring & 8.4 & 2.3 & 0.8 & 1.2 & 3.7 & 14.4 & 21.1 & 0.8 & 0.4 & 0.6 & 3.3 \\
\hline & Summer & 6.8 & 3.0 & 0.6 & 1.1 & 3.5 & 14.4 & 21.6 & 0.4 & 0.2 & 0.6 & 3.5 \\
\hline & Autumn & 10.9 & 2.4 & 0.6 & 1.1 & 7.3 & 18.4 & 28.8 & 0.5 & 0.3 & 0.7 & 3.5 \\
\hline & Winter & 11.4 & 1.5 & 0.6 & 0.8 & 11.8 & 19.7 & 33.7 & 0.1 & 0.4 & 0.8 & 3.8 \\
\hline \multirow[t]{4}{*}{ CTR } & Sprin & 4.2 & 2.3 & $\overline{0.3}$ & 1.1 & 0.3 & 3.3 & 6.3 & & 0.2 & 0.6 & 4.9 \\
\hline & Summer & 3.5 & 3.6 & 0.2 & 1.4 & 0.2 & 2.6 & 5.1 & 0.5 & 0.1 & 0.5 & 4.8 \\
\hline & Autumn & 6.5 & 2.7 & 0.2 & 1.2 & 0.6 & 5.8 & 8.9 & 0.4 & 0.2 & 0.6 & 4.1 \\
\hline & Winter & 6.9 & 1.5 & 0.3 & 0.8 & 0.8 & 7.5 & 11.1 & 0.6 & 0.4 & 0.6 & 3.5 \\
\hline \multirow[t]{4}{*}{ BHM } & Spring & 13.8 & 2.8 & 2.4 & 1.5 & 23.4 & 39.5 & 65.1 & 0.9 & 0.6 & 2.1 & 7.3 \\
\hline & Summer & 14.4 & 4.6 & 2.4 & 2.1 & 16.5 & 34.4 & 55.3 & 1.0 & 0.3 & 1.7 & 6.9 \\
\hline & Autumn & 15.4 & 2.8 & 2.1 & 1.4 & 32.9 & 39.2 & 73.1 & 0.5 & 0.4 & 1.8 & 5.8 \\
\hline & Winter & 13.3 & 1.7 & 1.4 & 1.1 & 40.2 & 39.8 & 81.2 & 0.4 & 1.0 & 1.7 & 5.4 \\
\hline \multirow[t]{4}{*}{ YRK } & Spring & 7.4 & 2.7 & 1.7 & 1.5 & 0.5 & 6.0 & 10.8 & 1.5 & 0.5 & 0.6 & 5.0 \\
\hline & & 7.0 & 4.6 & 2.5 & 2.3 & 0.4 & 5.7 & 9.6 & & 0.3 & & 5.7 \\
\hline & Autumn & 8.1 & 3.0 & 2.6 & 1.6 & 0.9 & 9.2 & 13.7 & 1.1 & 0.5 & 0.6 & 4.4 \\
\hline & Winter & 10.9 & 1.5 & 2.0 & 1.1 & 1.6 & 13.0 & 17.7 & 1.0 & 1.0 & 0.6 & 3.5 \\
\hline \multirow[t]{4}{*}{ JST } & Spring & 11.9 & 2.7 & 1.3 & 1.5 & 34.9 & 43.3 & 80.8 & 1.2 & 0.5 & 1.2 & 6.3 \\
\hline & Summer & 10.9 & 4.7 & 1.2 & 2.2 & 17.0 & 40.1 & 61.5 & 1.2 & 0.3 & 1.4 & 6.1 \\
\hline & Autumn & 12.1 & 3.0 & 1.2 & 1.6 & 49.3 & 44.2 & 96.6 & 0.7 & 0.5 & 1.5 & 5.7 \\
\hline & Winter & 15.8 & 1.6 & 1.0 & 1.2 & 60.3 & 47.9 & 107.7 & 0.5 & 1.1 & 1.4 & 6.2 \\
\hline
\end{tabular}

a. Winter $=$ Dec - Feb; Spring $=$ Mar - May; Summer $=$ Jun - Aug; Autumn $=$ Sep - Nov 
Table S4. Comparisons of rural, summertime - midday NMOC mean concentrations from sites in the Southeast. Units are ppbC except as indicated. Intercomparison of NMOC concentrations indicates that isoprene is the major NMOC in the rural Southeast even during the period of maximum photochemical processing.

\begin{tabular}{|c|c|c|c|c|c|c|c|c|}
\hline Species & $\begin{array}{c}\text { West } \\
\text { Alabama }^{\text {a }} \\
(1990)\end{array}$ & $\begin{array}{c}\text { Oak } \\
\text { Grove, } \\
\text { MS }^{b} \\
(1994)\end{array}$ & $\begin{array}{c}\text { Centreville, } \\
\text { AL }^{\mathrm{b}} \\
(1994)\end{array}$ & $\begin{array}{c}\text { Yorkville, } \\
\text { GA }^{\mathrm{b}} \\
(1994)\end{array}$ & $\begin{array}{c}\text { Yorkville, } \\
\text { GA }^{\mathrm{c}} \\
(2002)\end{array}$ & $\begin{array}{c}\text { Yorkville, } \\
\text { GA }^{c} \\
(2011)\end{array}$ & $\begin{array}{c}\text { Nashville, } \\
\text { TN } \\
\text { (rural) }^{\mathrm{d}} \\
(1995)\end{array}$ & $\begin{array}{c}\text { Relative } \\
\text { OH } \\
\text { Reactivity }\end{array}$ \\
\hline ethane & - & 1.02 & 1.74 & 0.96 & 1.73 & 3.23 & nd & 41 \\
\hline ethene & - & 0.57 & 0.61 & 0.90 & 0.14 & 0.25 & nd & 3 \\
\hline acetylene & - & 0.45 & 0.36 & 0.72 & 0.08 & 0.20 & nd & low \\
\hline propane & 3.6 & 4.04 & 2.60 & 1.89 & 1.68 & 2.02 & - & 14 \\
\hline propene & - & 0.61 & 0.73 & 1.61 & 0.02 & 0.52 & - & 2 \\
\hline i-butane & 0.8 & 1.25 & 0.77 & 0.91 & 0.20 & 0.33 & 0.44 & 29 \\
\hline n-butane & 1.2 & 1.87 & 1.48 & 1.96 & 0.81 & 0.76 & 0.76 & 17 \\
\hline i-pentane & 1.3 & 5.90 & 2.22 & 5.02 & 1.35 & 0.95 & 0.95 & 10 \\
\hline n-pentane & 0.45 & 16.5 & 1.04 & 2.15 & 0.49 & 0.60 & 0.38 & 23 \\
\hline isoprene & $31\left(4.5^{\mathrm{g}}\right)$ & 11.2 & 21.2 & 9.80 & 6.54 & 9.62 & 4.25 & 1 \\
\hline $\begin{array}{l}\text { 2-methyl- } \\
\text { pentane }\end{array}$ & 0.27 & 2.16 & 3.34 & 3.92 & 0.17 & 0.12 & 0.25 & 25 \\
\hline $\begin{array}{l}\text { 3-methyl- } \\
\text { pentane }\end{array}$ & 0.13 & 0.93 & 0.82 & 1.05 & 0.07 & 0.17 & 0.09 & 34 \\
\hline cyclopentane & - & 0.16 & 0.05 & 0.19 & 0.0 & 0.03 & - & 57 \\
\hline n-hexane & 0.12 & 0.72 & 0.68 & 0.68 & 0.07 & 0.36 & - & 28 \\
\hline $\begin{array}{l}\text { 2-methyl-2- } \\
\text { butene }\end{array}$ & - & 0.10 & 0.06 & 0.19 & - & - & - & 5 \\
\hline benzene & 0.60 & 0.69 & 0.61 & 1.22 & 0.21 & 0.22 & 1.26 & 52 \\
\hline toluene & 0.52 & 3.63 & 1.20 & 4.75 & 0.77 & 0.62 & 0.56 & 10 \\
\hline $\begin{array}{l}\text { methyl- } \\
\text { cyclohexane }\end{array}$ & 0.056 & 0.69 & 0.42 & 0.57 & 0.00 & 0.01 & - & \\
\hline $\mathrm{m}, \mathrm{p}$-xylene & 0.16 & 0.89 & 0.40 & 1.80 & 0.12 & 0.15 & $\begin{array}{c}0.024- \\
0.072\end{array}$ & 4 \\
\hline o-xylene & 0.072 & 0.36 & 0.13 & 0.73 & 0.03 & 0.03 & 0.048 & 19 \\
\hline ethyl benzene & 0.08 & 0.45 & 0.20 & 0.95 & 0.04 & 0.08 & 0.14 & 31 \\
\hline$\alpha$-pinene & 3.0 & 2.71 & 1.60 & 0.11 & - & - & 0.07 & 8 \\
\hline$\beta$-pinene & 1.7 & 3.04 & 1.36 & 2.06 & - & - & 0.06 & 42 \\
\hline acetone $^{\mathrm{f}}$ & 4.2 & - & - & - & - & - & 3.83 & \\
\hline acetaldehyde $\mathrm{f}^{\mathrm{f}}$ & 1.3 & - & - & - & - & - & 1.56 & \\
\hline $\begin{array}{l}\text { methyl-ethyl- } \\
\text { ketone }^{f}\end{array}$ & 0.49 & - & - & - & - & - & 0.30 & \\
\hline ethanol $^{\mathrm{f}}$ & 1.2 & - & - & - & - & - & 0.38 & \\
\hline
\end{tabular}

${ }^{\mathrm{a} D}$ Data June-July of 1990 1:600 h CST for Kinterbish, Alabama from Goldan et al. (1995).

b Data summer 1994 12:00-13:00 h local standard time as part of SOS from Hagerman et al. (1997).

${ }^{c}$ Data from PAMS archive for YRK 2002 and 2011, 10:00-15:00 h EST.

${ }^{\mathrm{d}}$ Data for summer daytime rural site $32 \mathrm{~km} \mathrm{SE}$ of Nashville, Tennessee 10:00-20:00 h local standard time from Riemer et al. (1998)

${ }^{\mathrm{e}}$ Ranking of $\mathrm{k}_{\mathrm{OH}}$ from highest to lowest?

${ }^{\mathrm{f}}$ Data in ppbv.

${ }^{\mathrm{g}}$ At 04:00 h CST 


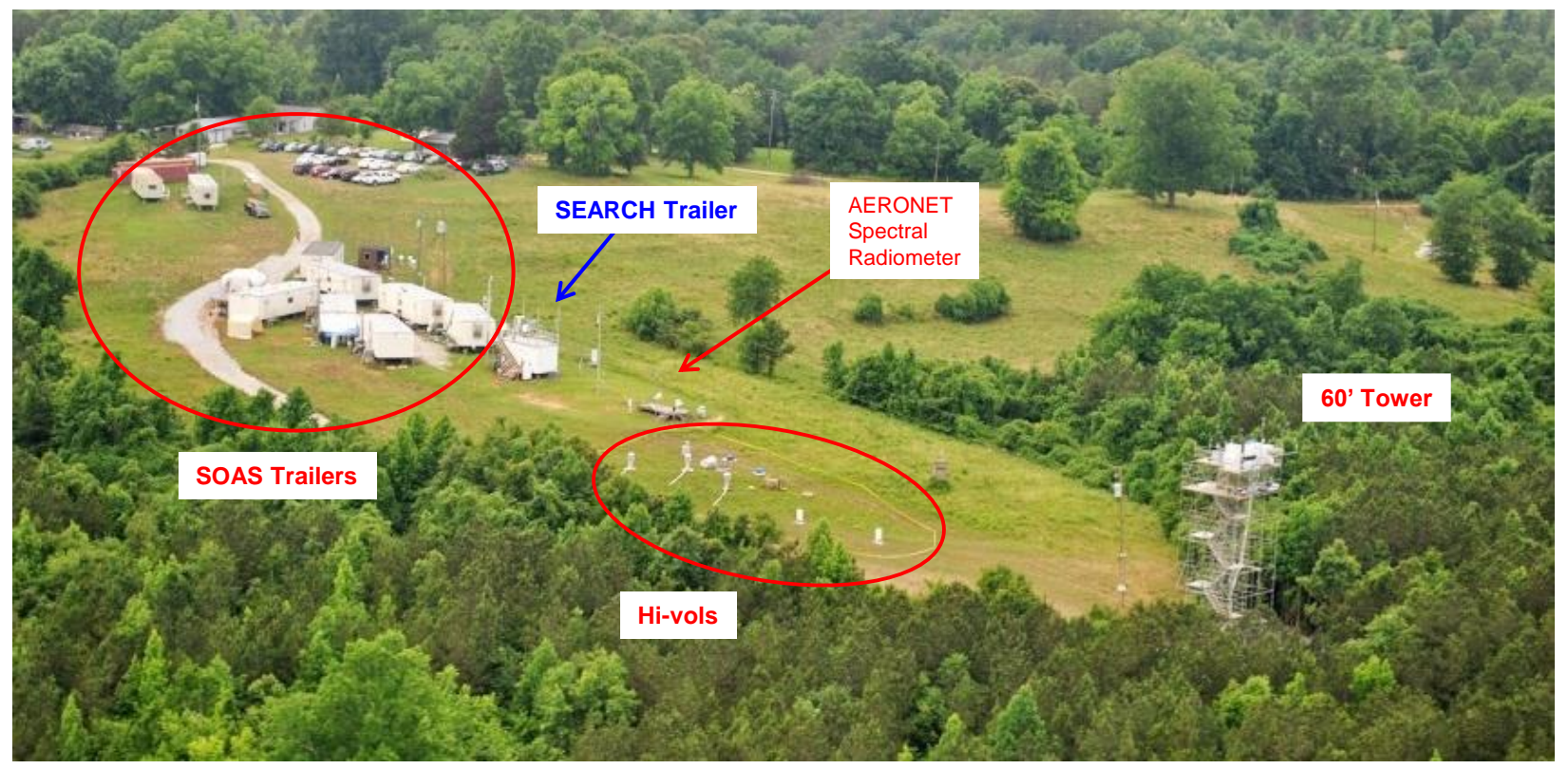

Figure S1. CTR site with instrumentation during the SOAS experiments in June-July, 2013. 

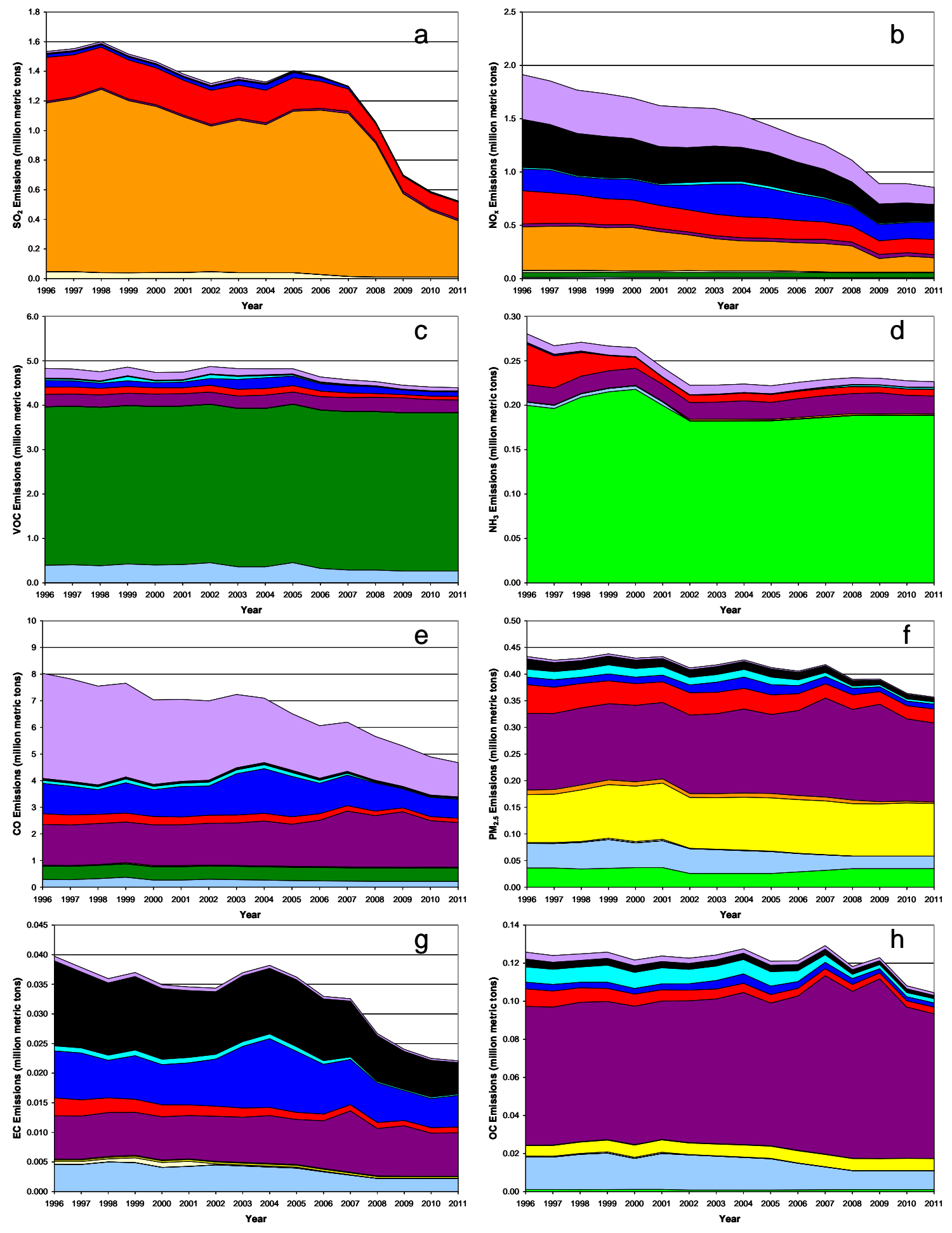

\begin{tabular}{|c|c|c|c|c|c|}
\hline $\begin{array}{l}\square \text { Agriculture } \\
\square \text { Fires }\end{array}$ & $\begin{array}{l}\square \text { Area } \\
\square \text { Industrial }\end{array}$ & $\begin{array}{l}\square \text { Vegetation\&soil } \\
\text { Nonroad }\end{array}$ & $\begin{array}{l}\square \text { Commercial } \\
\square \text { Residential }\end{array}$ & $\begin{array}{l}\square \text { Dust } \\
\text { On-road diesel }\end{array}$ & $\begin{array}{l}\square \text { EGU } \\
\square \text { On-road gas }\end{array}$ \\
\hline
\end{tabular}

Figure S2. Emission trends in the study domain (Blanchard et al., 2013c). (a) $\mathrm{SO}_{2}$, (b) $\mathrm{NO}_{\mathrm{x}}$, (c) VOC, (d) $\mathrm{NH}_{3}$, (e) CO, (f) $\mathrm{PM}_{2.5}$, (g) EC, (h) POC. 


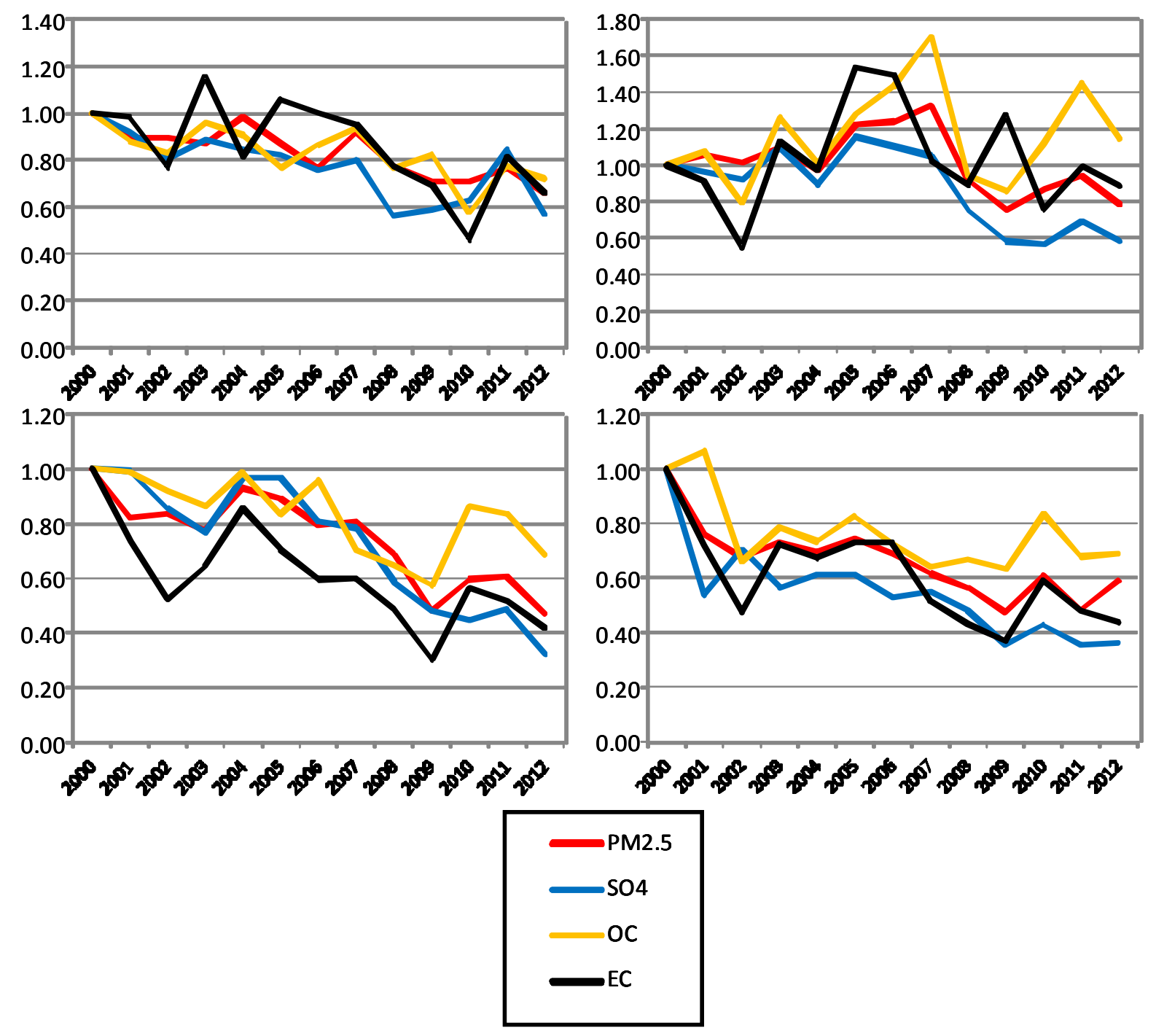

Figure S3. Normalized trends by season for $\mathrm{PM}_{2.5}, \mathrm{SO}_{4}$, OC and EC, 2000-2012. Q1 (Jan - Mar) is upper left, Q2 upper right, Q3 lower left, and Q4 lower right. All concentrations are normalized to year 2000 . 


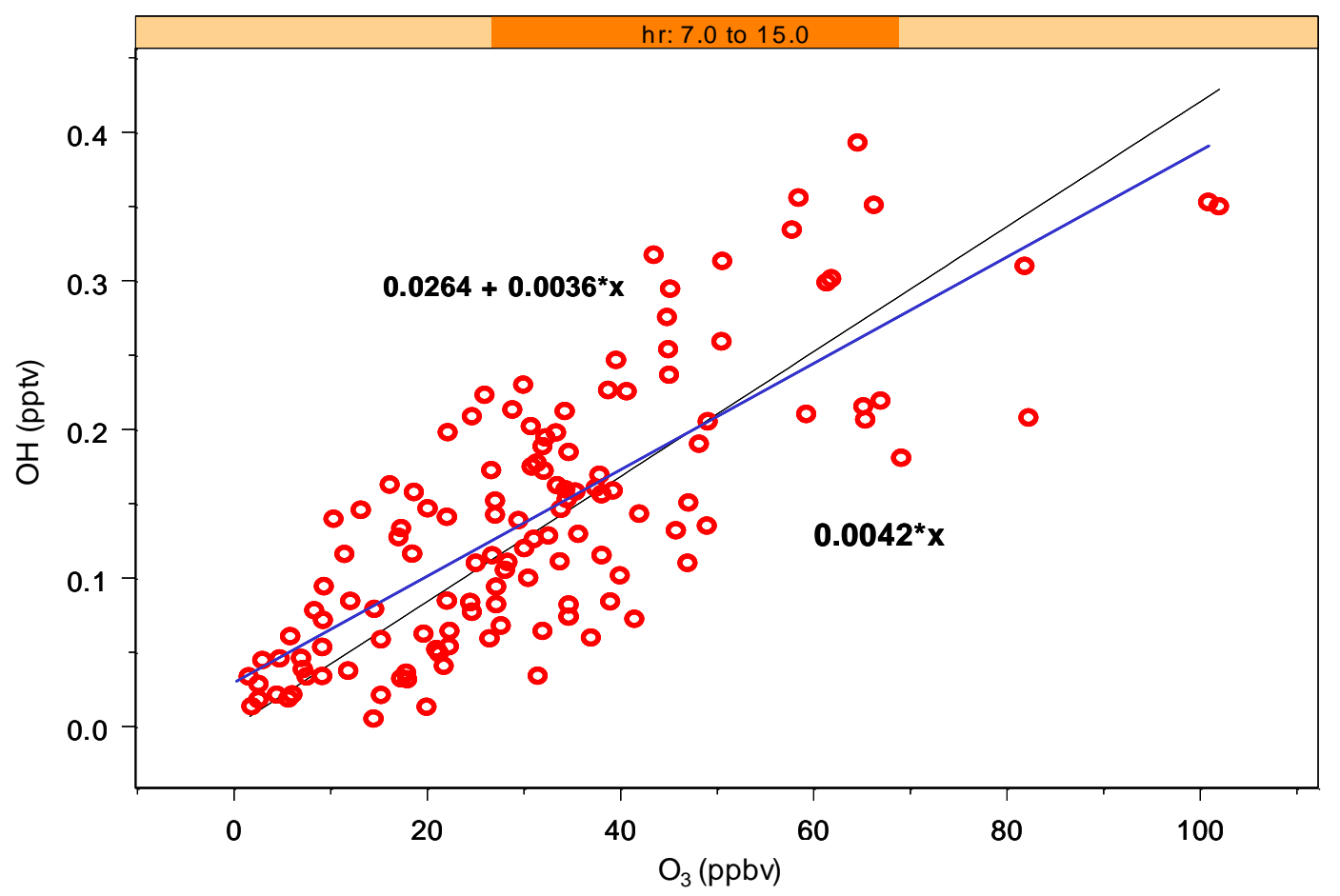

Figure S4. Daytime relation measured relationship between $\mathrm{OH}$ and $\mathrm{O}_{3}$ found in New York City. Lines indicate regressions with (blue) and without (black) intercept. (From K. Demerjian, New York supersite program, summer 2001; personal communication, and including data derived from measurements of Ren et al., 2003). 

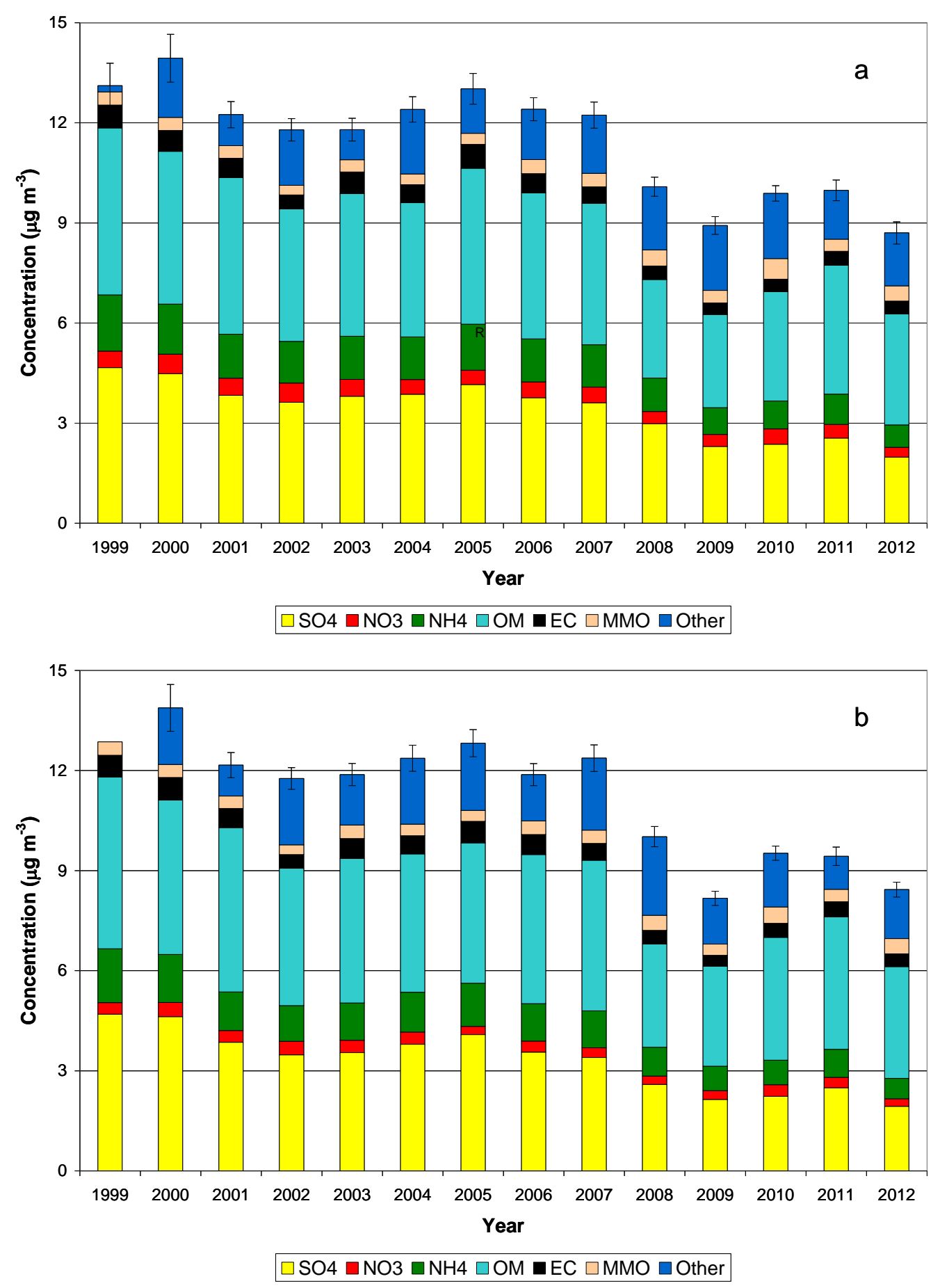

Figure S5. Trends in annual $\mathrm{PM}_{2.5}$ mass concentration and composition at (a) rural SEARCH sites (mean) and (b) CTR. "OM" is $1.4 * \mathrm{OC}$. "Other" is the difference between federal reference method (FRM) mass concentrations and the sum of measured components; it includes retained water, unmeasured species, and organic mass exceeding $1.4 * \mathrm{OC} ; \mathrm{NO}_{3}$ is $\mathrm{pNO}_{3}$. Uncertainties are one standard error of the intersite mean FRM mass concentrations. $\mathrm{OM}$ and $\mathrm{SO}_{4}$ are the major contributors to annual $\mathrm{PM}_{2.5}$ mass concentration over the entire period; both are declining, but the $\mathrm{SO}_{4}$ decrease is stronger. 

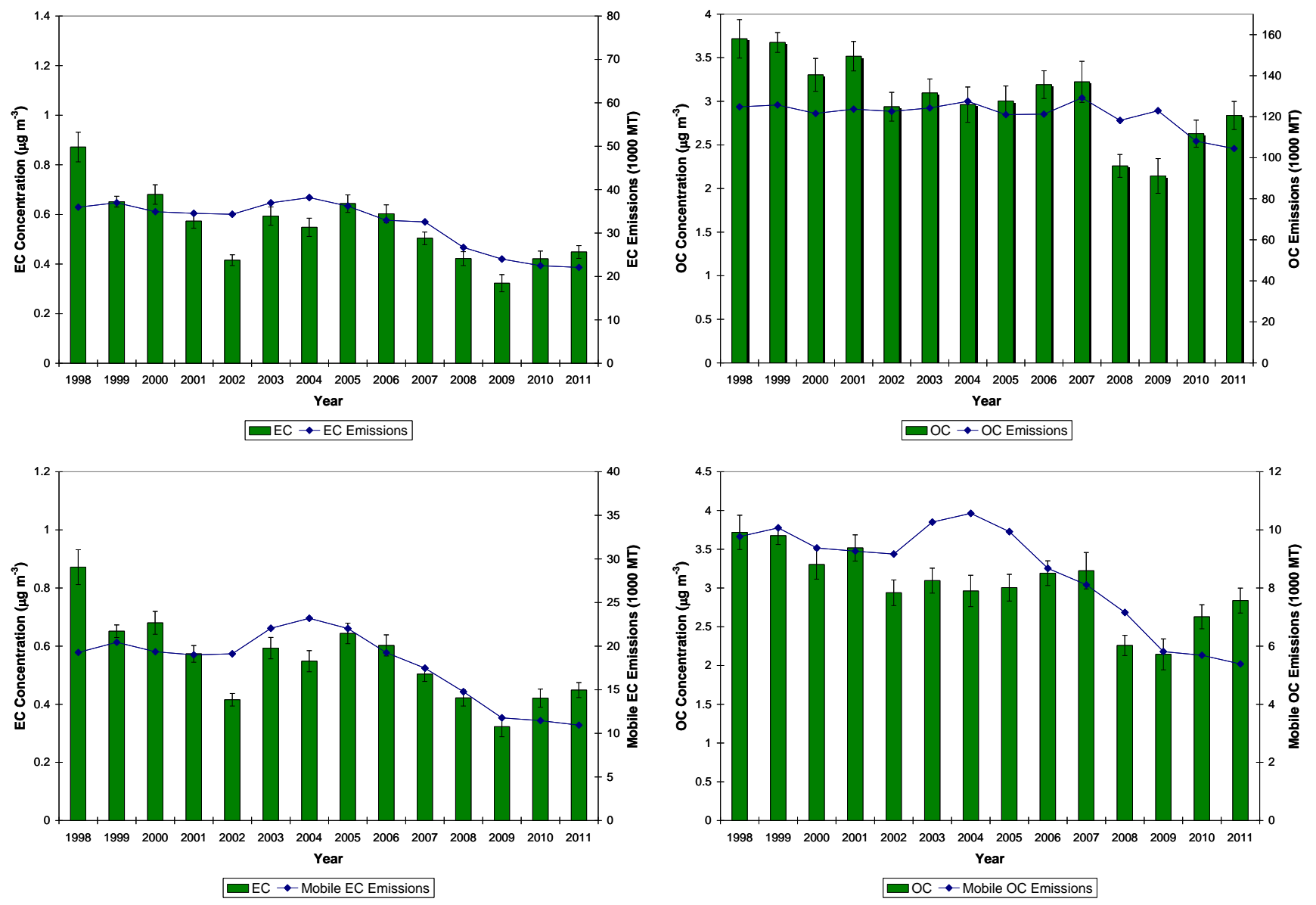

Figure S6. Mean annual EC and OC concentrations at CTR compared with emissions of EC and OC from plus non-road) sources (bottom) within Alabama, Georgia, Northwest Florida, and Mississippi. Emissions data based on the (US) National Emissions Inventory are from Blanchard et al., (2013c). EC follows closely the estimates of total and mobile emission trends, while OC corresponds less to total emissions and more to mobile emissions (see also Table 1 and discussion in text). 

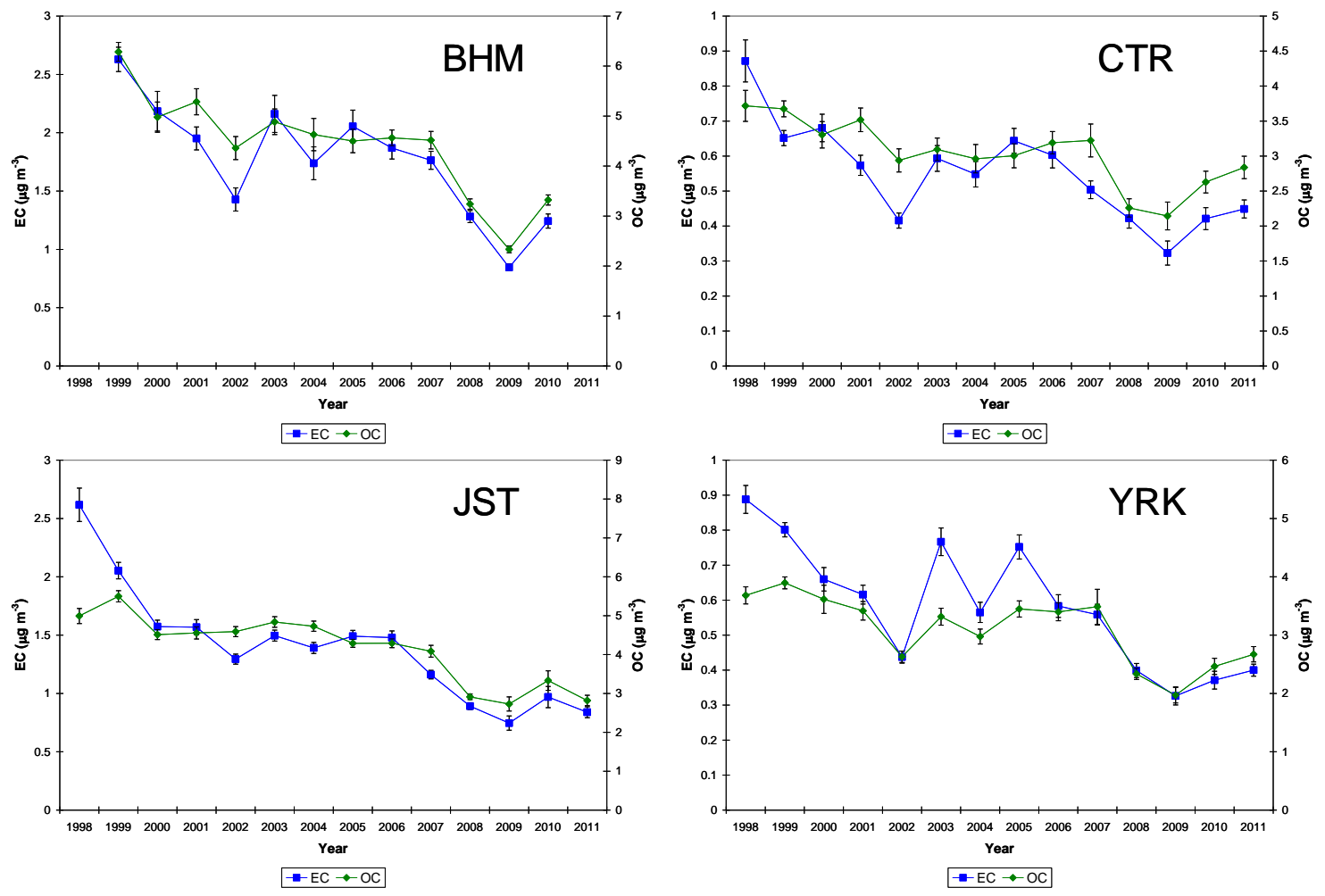

Figure S7. Mean annual concentrations of EC and OC at SEARCH sites. The parallel decrease in mean concentrations indicates common origins of the carbon components. 

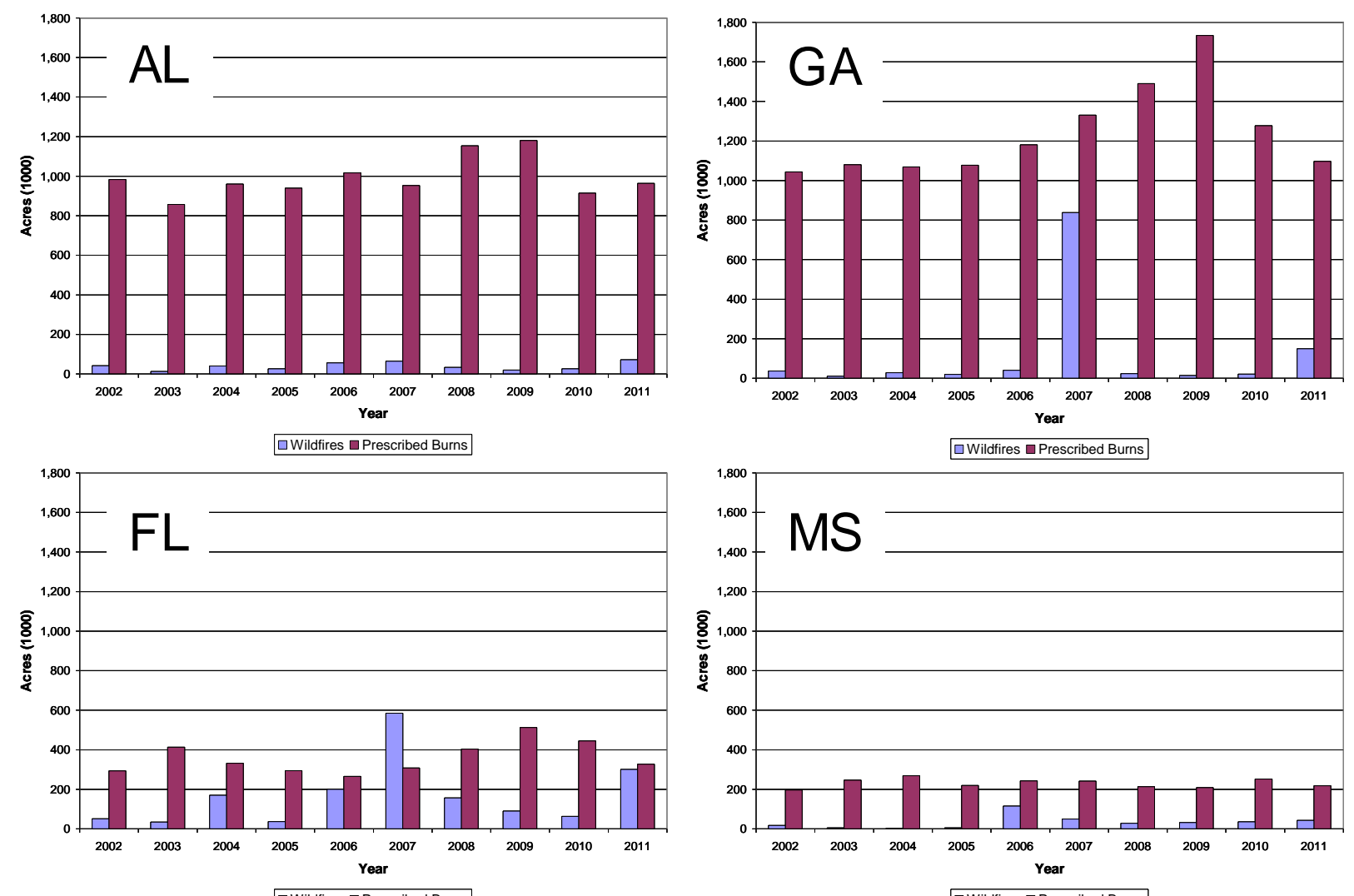

$\square$ Wildfires $\square$ Prescribed Burns

口Wildfires घPrescribed Burns

Figure S8. Annual wildfire and prescribed burn acreage by state, 2002-2011. The annual prevalence of prescribed fires is an important factor in rural ambient EC and OC concentrations. Alabama and Georgia data represent all prescribed burns and wildfire. Florida and Mississippi data represent all wildfires and prescribed burns by federal agencies. Total annual prescribed burn acreage averages approximately 2 million acres in Florida and 200,000 to 500,000 acres in Mississippi. Source: Blanchard et al. (2013c). 

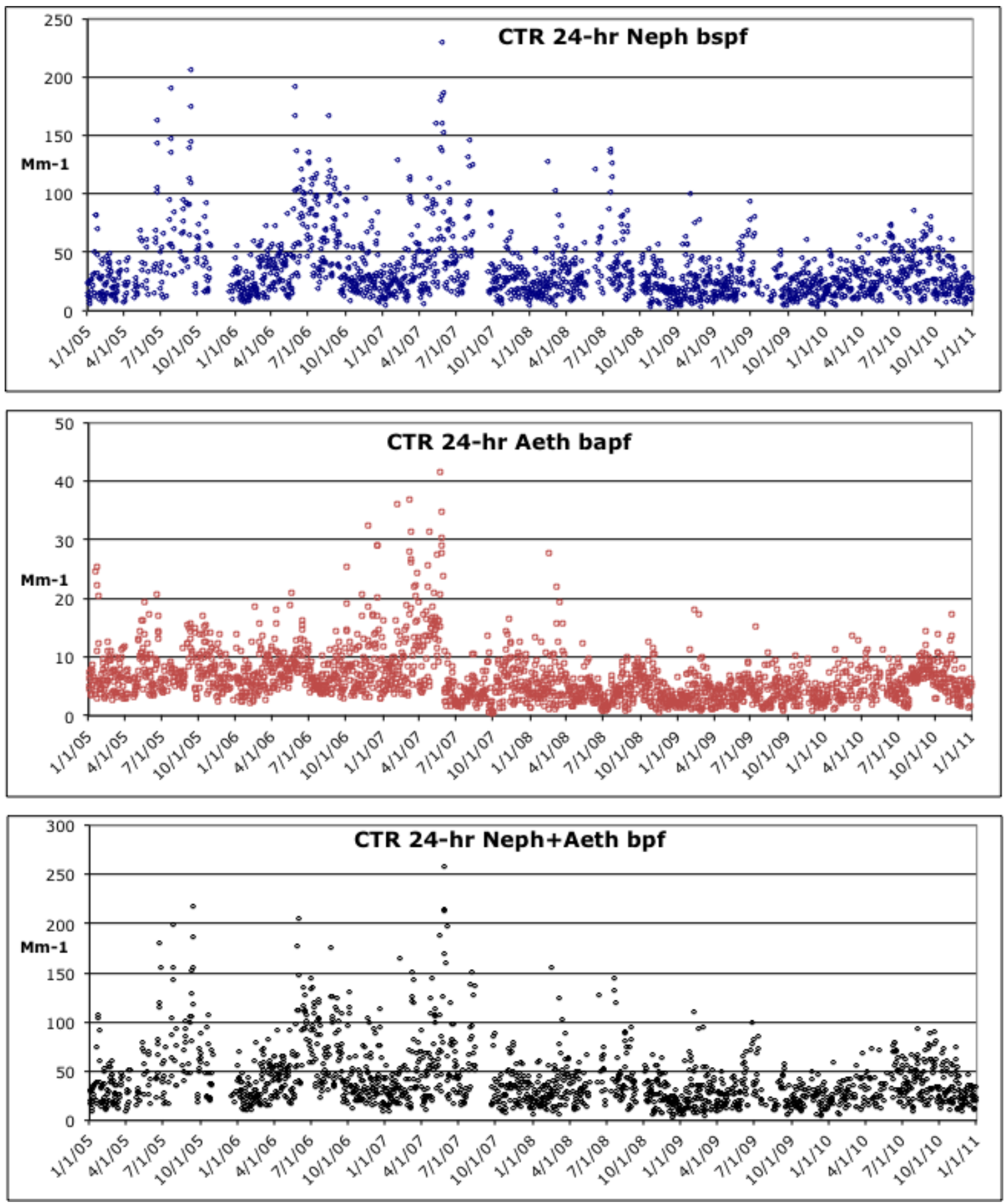

Figure S9. Timelines of 24-hr average light scattering by desiccated fine particles (top plot), light absorption by those particles (middle plot), and their sum (bottom plot) at CTR during the six years 2005-10. 

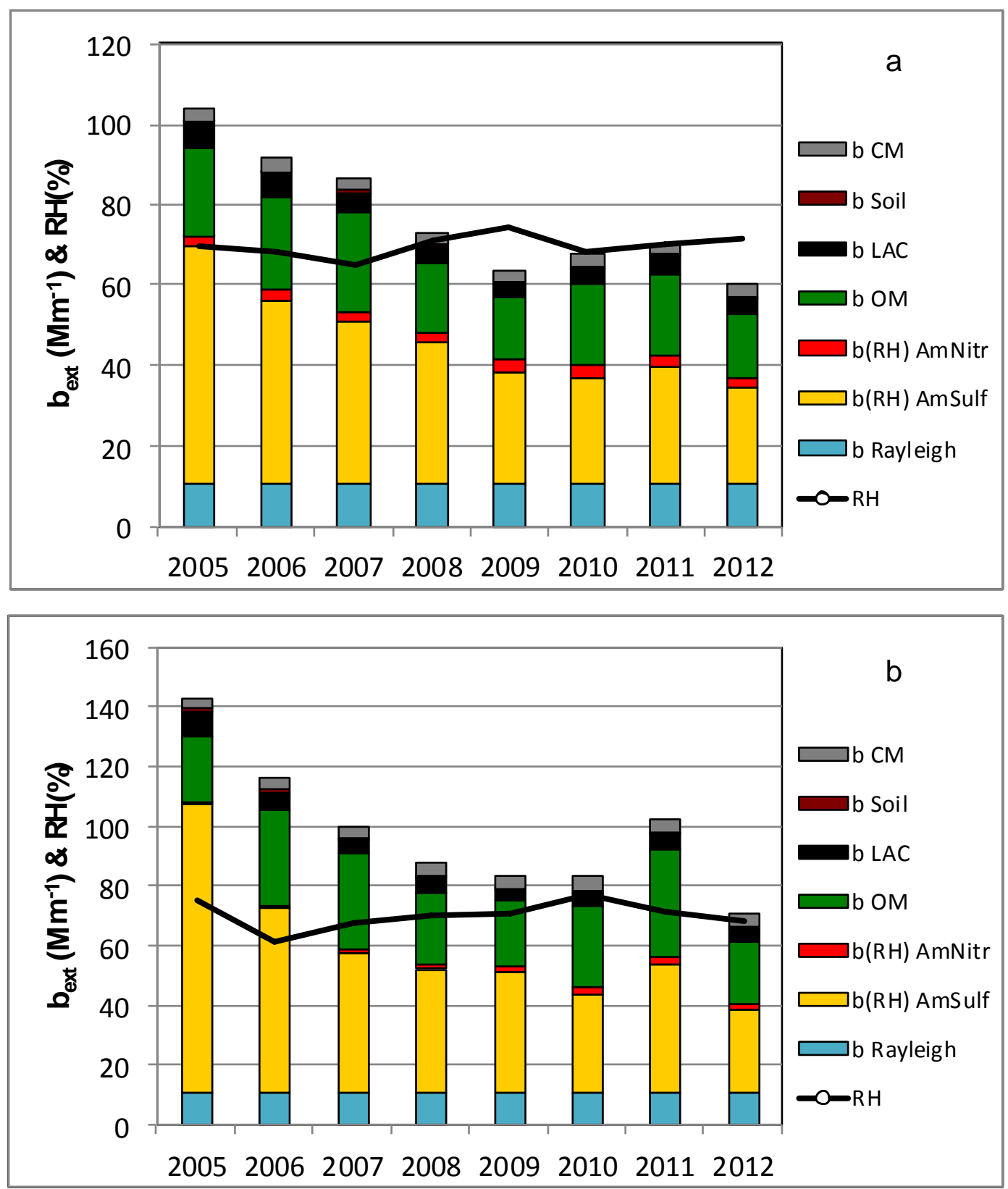

Figure S10. (a) Annual averages of light extinction by component at CTR from 2005 to 2012, as calculated using the new IMPROVE algorithm (Pitchford et al., 2007). (b) Period (June 1-July 15) averages of light extinction by component at CTR for each year. The contributions to light extinction include RH-dependent factors for ammonium sulfate and ammonium nitrate. The black line shows the average measured RH during each year. 
a. GA

b. AL

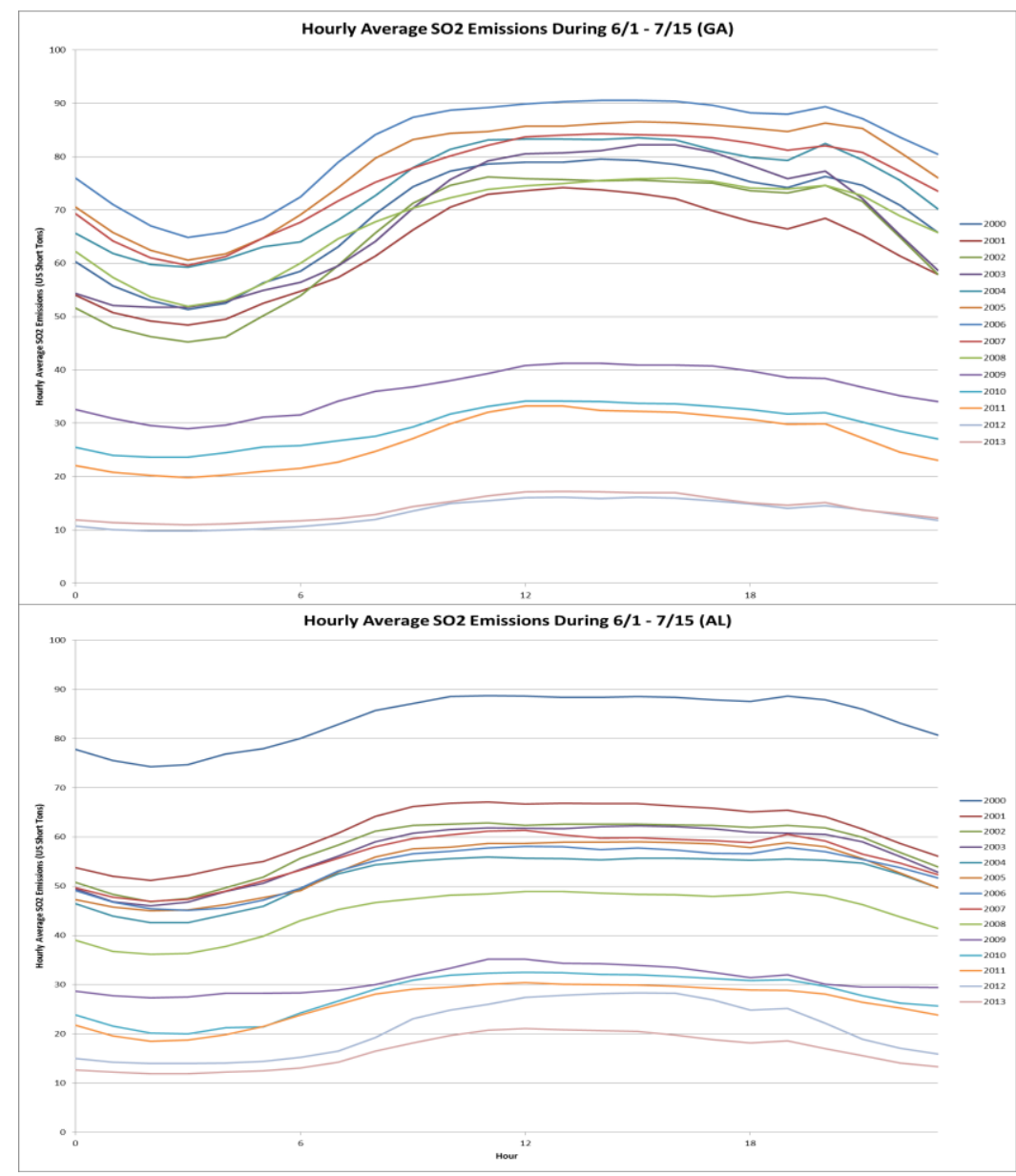

c. MS

Hourly Average SO2 Emissions During 6/1 - 7/15

(MS)

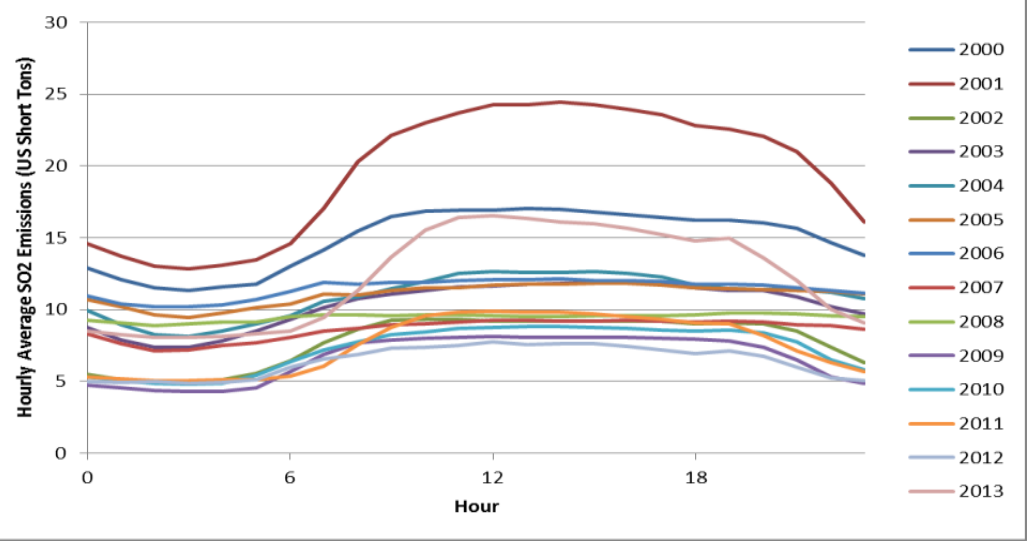

Figure S11a. Diurnal (LST) variation in $\mathrm{SO}_{2}$ emissions from electric generating units in three states, Alabama, Georgia and Mississippi. Trends between 2000 and 2012 from the Air Markets Program Data (AMPD) (http://ampd.epa.gov/ampd). 
a. GA

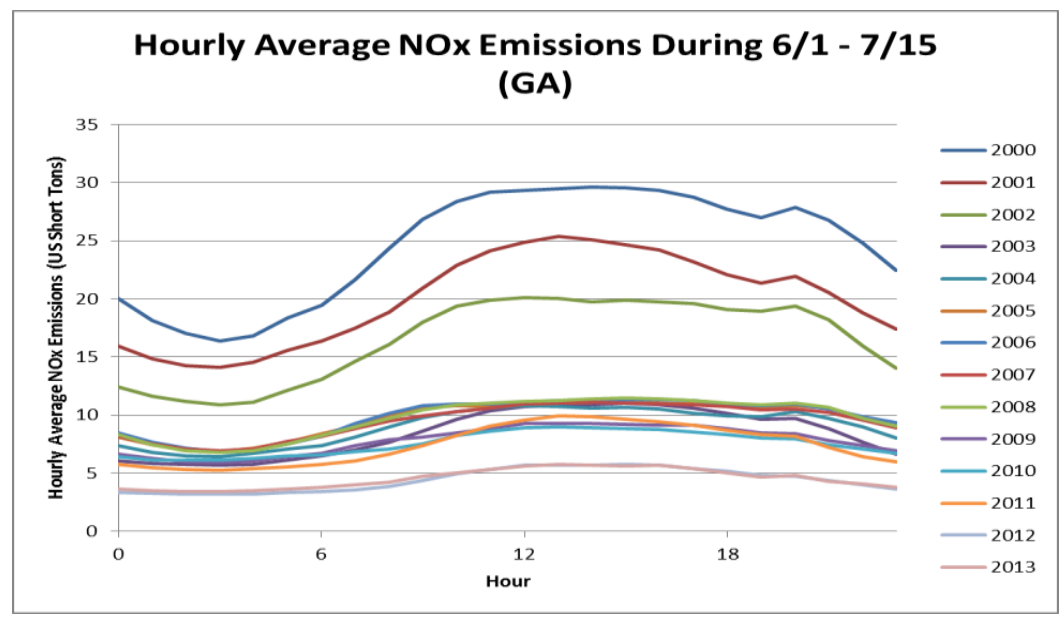

b. AL

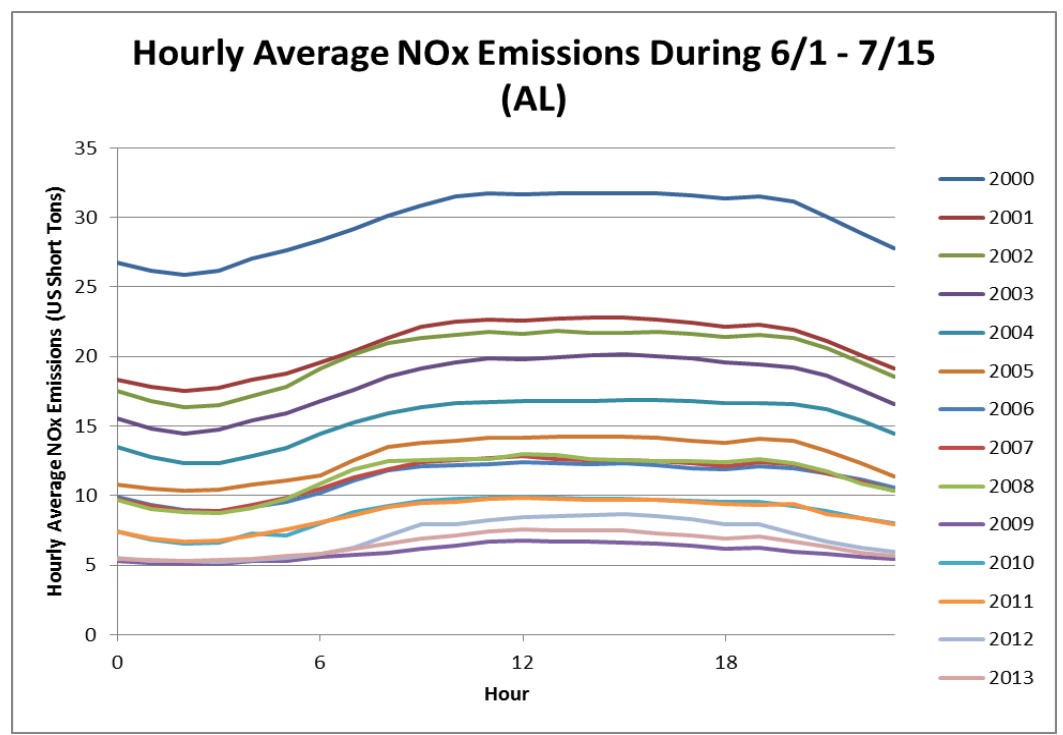

c. MS

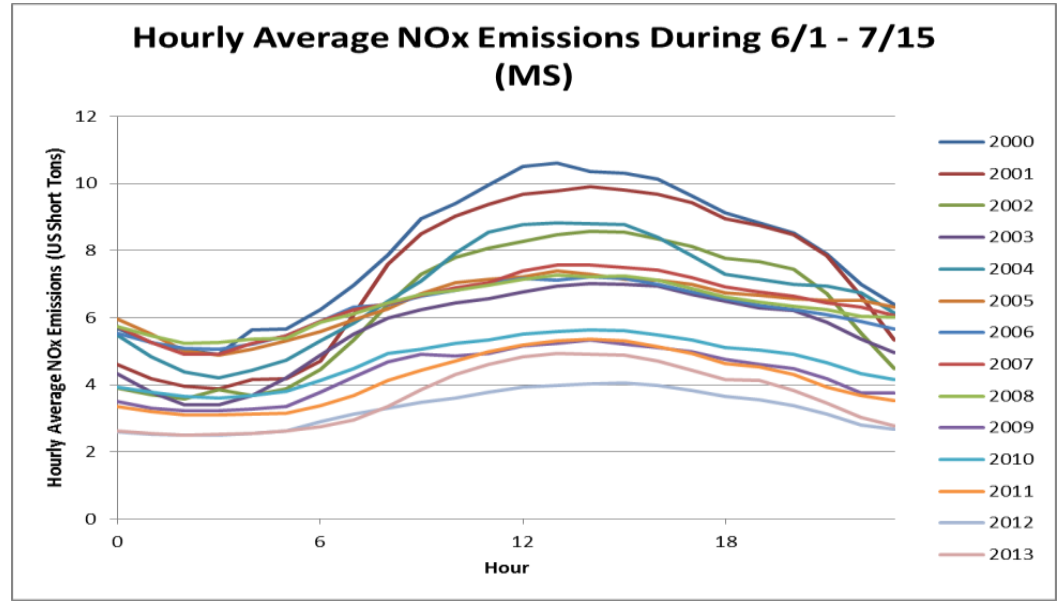

Figure S11b. Diurnal (LST) variation in $\mathrm{NO}_{\mathrm{x}}$ emissions from electric generating units in three states, Alabama, Georgia and Mississippi. Trends between 2000 and 2012 from the Air Markets Program Data (AMPD) (http://ampd.epa.gov/ampd). 


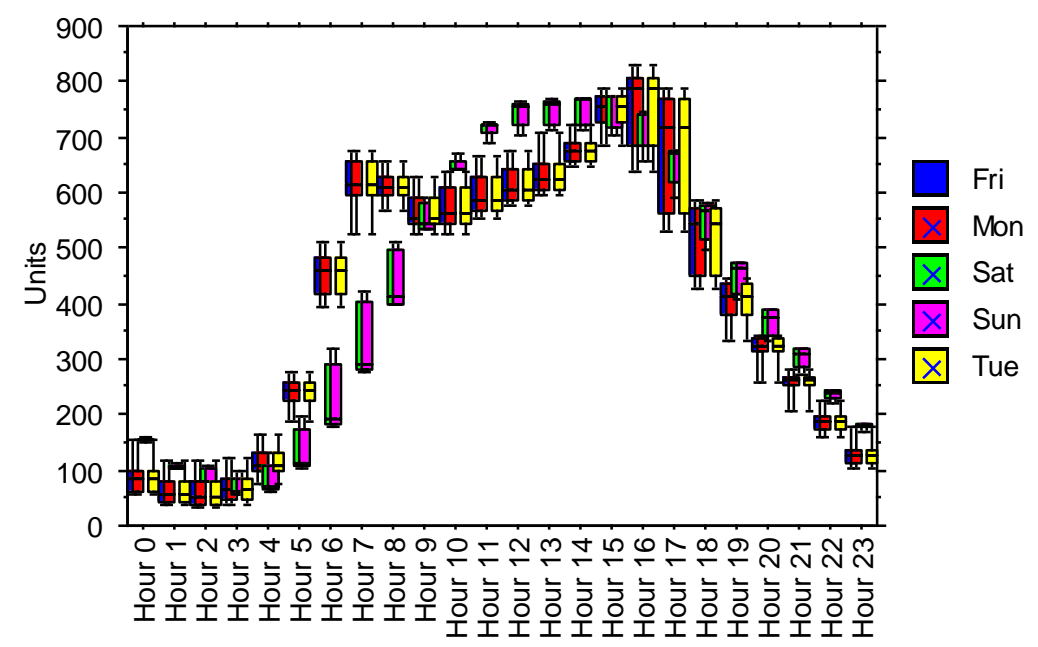

Figure S12. Statistical LST distributions of national-average hourly allocations of mobile-source emissions across 48 vehicle categories showing differences among days of the week. The "Tuesday" profile applies also to Wednesdays and Thursdays. Each box-and-whiskers indicates the $5^{\text {th }}, 25^{\text {th }}, 50^{\text {th }}, 75^{\text {th }}$, and $95^{\text {th }}$ percentiles of the 48 vehicle categories for a given day and hour. Units are arbitrary and sum to approximately 10,000 across 24 hours. Source: U.S. EPA, ftp://ftp.epa.gov/EmisInventory/2011v6/v1platform/reports/temporal_profiles/, http://www.cmascenter.org/smoke/documentation/3.5/html/ch08s03.html (accessed February 28, 2014). 

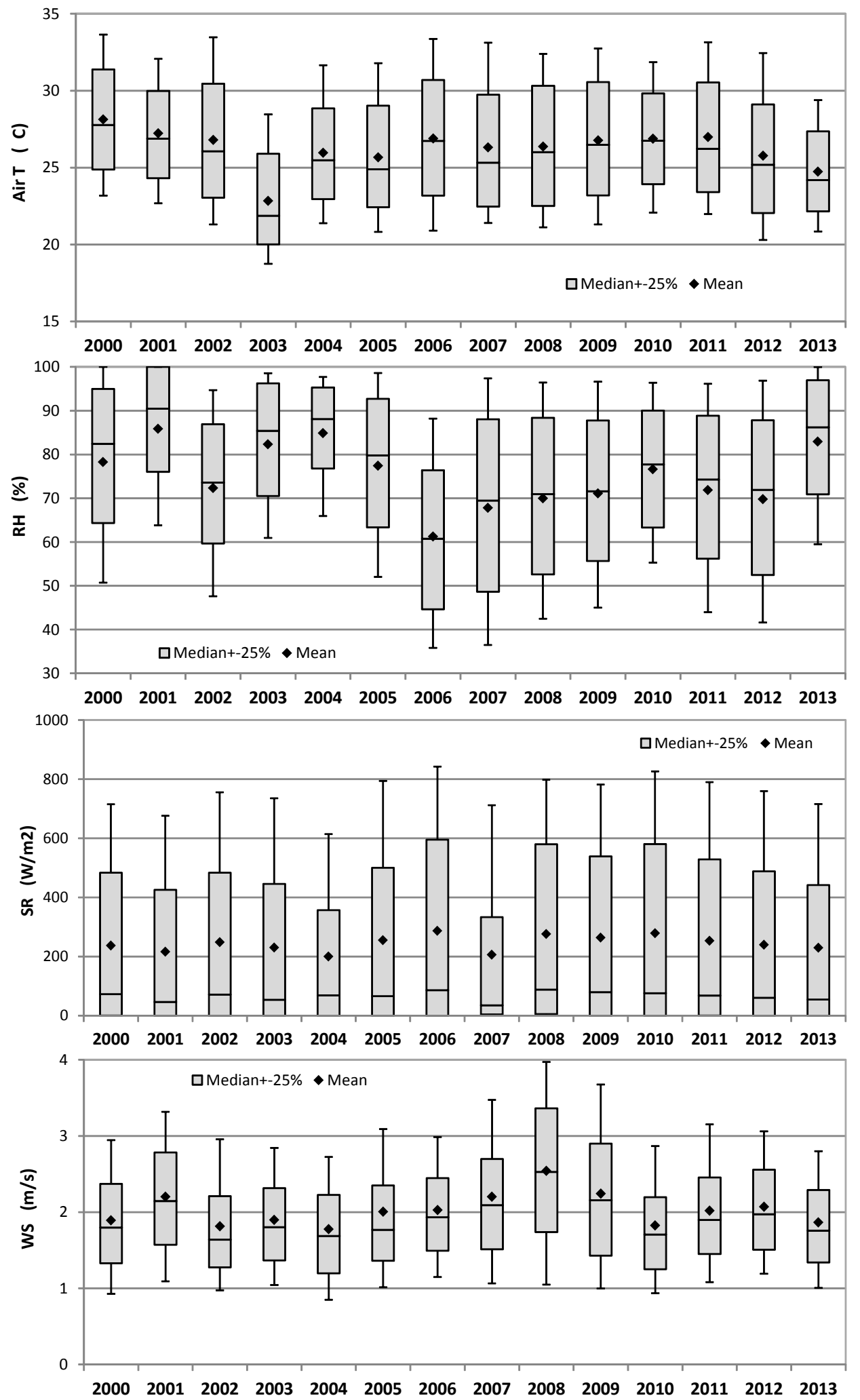

Figure S13. Statistical distributions of hourly temperature (T), relative humidity $(\mathrm{RH})$, solar radiation (SR), and wind speed (WS) at CTR from June 1 through July 15 each year. The plots indicate the median (horizontal line), mean (diamonds), $25^{\text {th }}$ and $75^{\text {th }} \%$ tiles (boxes), and the $10^{\text {th }}$ and $90^{\text {th }}$ percentiles (vertical lines). 

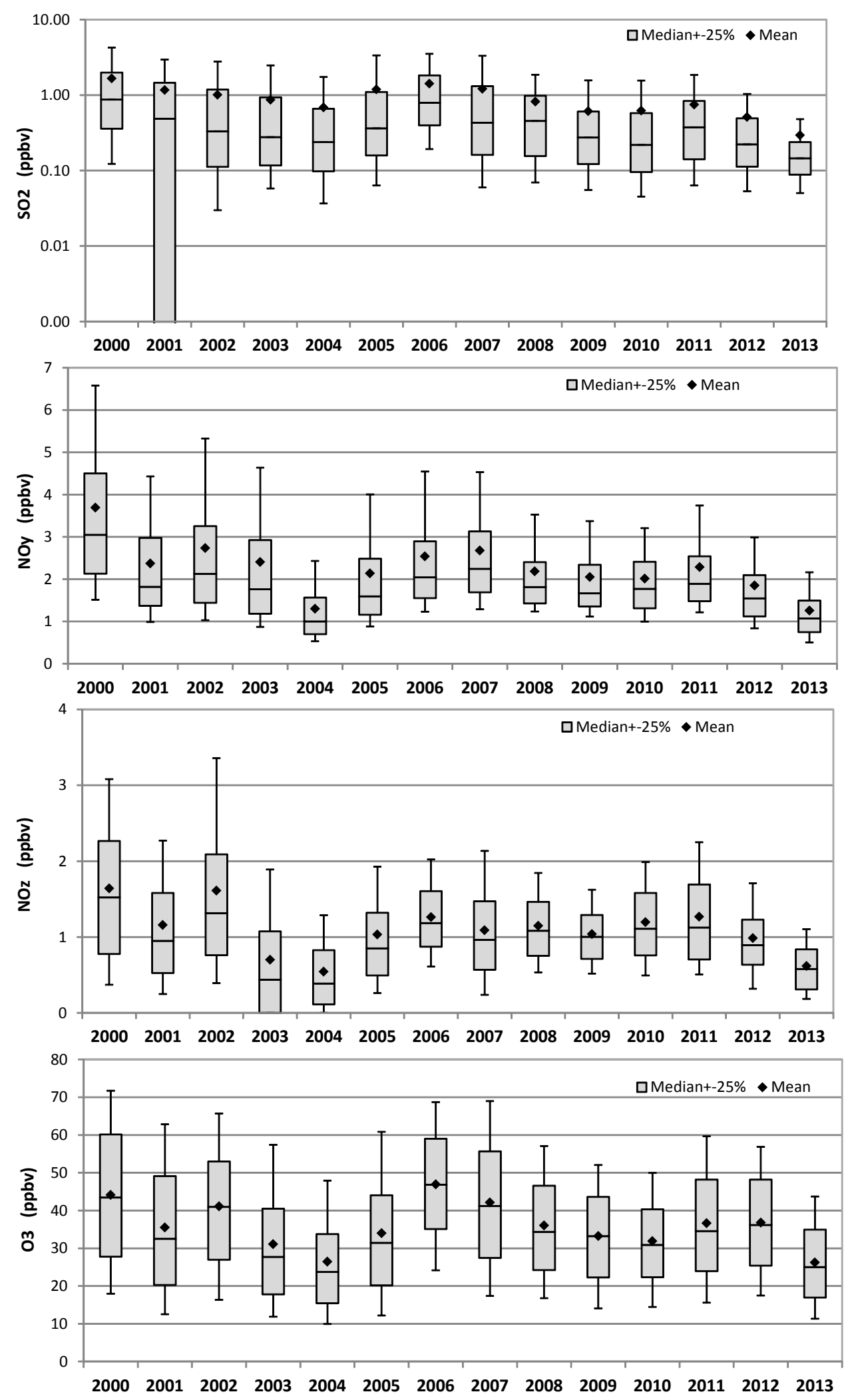

Figure S14. Statistical distributions of trace gases $\mathrm{SO}_{2}, \mathrm{NO}_{\mathrm{y}}, \mathrm{NO}_{z}$, and $\mathrm{O}_{3}$ at CTR from June 1 through July 15 each year. The plots indicate the median (horizontal line), mean (diamonds), $25^{\text {th }}$ and $75^{\text {th }} \%$ tiles (boxes), and the $10^{\text {th }}-90^{\text {th }}$ percentiles (vertical lines). 

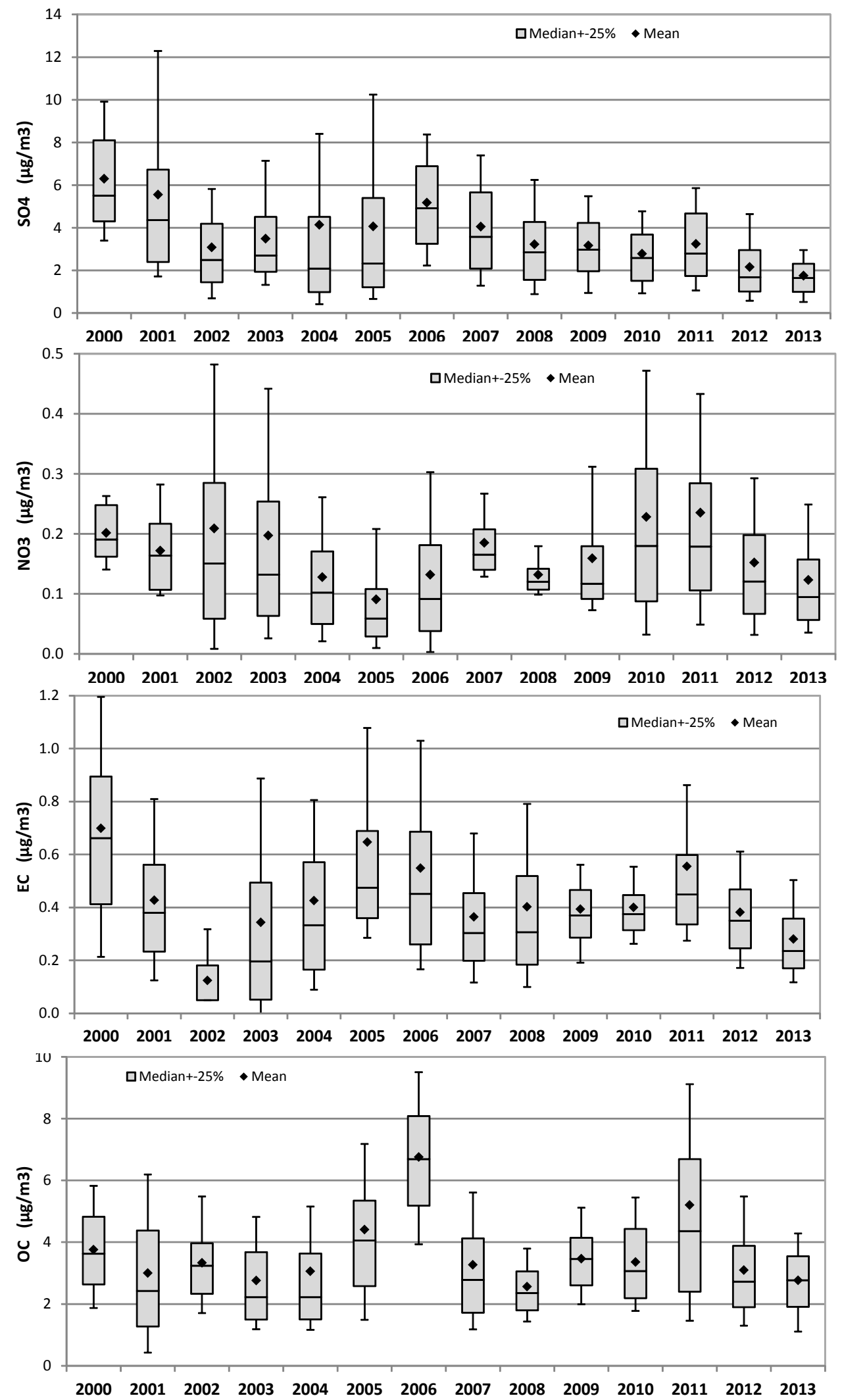

Figure S15. Statistical distributions of $\mathrm{PM}_{2.5} \mathrm{SO}_{4}, \mathrm{NO}_{3}, \mathrm{EC}$, and OC at CTR from June 1 through July 15 each year. The plots indicate the median (horizontal line), mean (diamonds), $25^{\text {th }}$ and $75^{\text {th }} \%$ tiles (boxes), and the $10^{\text {th }}-90^{\text {th }}$ percentiles (vertical lines). 


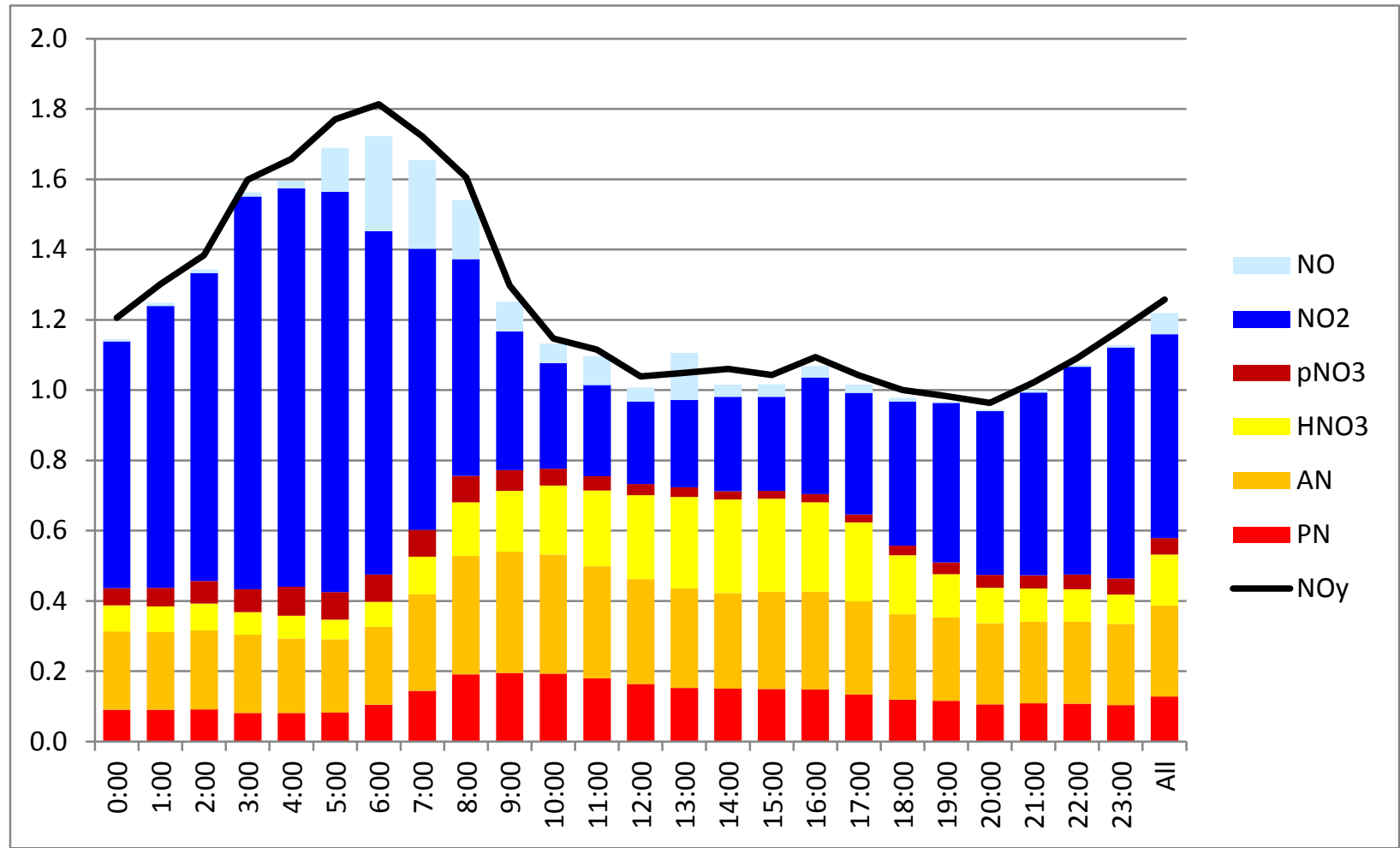

Figure S16. Composite ambient odd-nitrogen balance for CTR June-July, 2013, showing mean hourly (local time) concentrations of $\mathrm{NO}, \mathrm{NO}_{2}, \mathrm{PM}_{2.5} \mathrm{NO}_{3}\left(\mathrm{pNO}_{3}\right), \mathrm{HNO}_{3}$, alkyl nitrates (AN), and peroxyalkylnitrates (PN) in relation to measured concentrations of $\mathrm{NO}_{\mathrm{y}}$. Alkyl nitrates (AN) and peroxyalkyl nitrates (PN) determined from thermophotolytic conversion to NO measurement. 


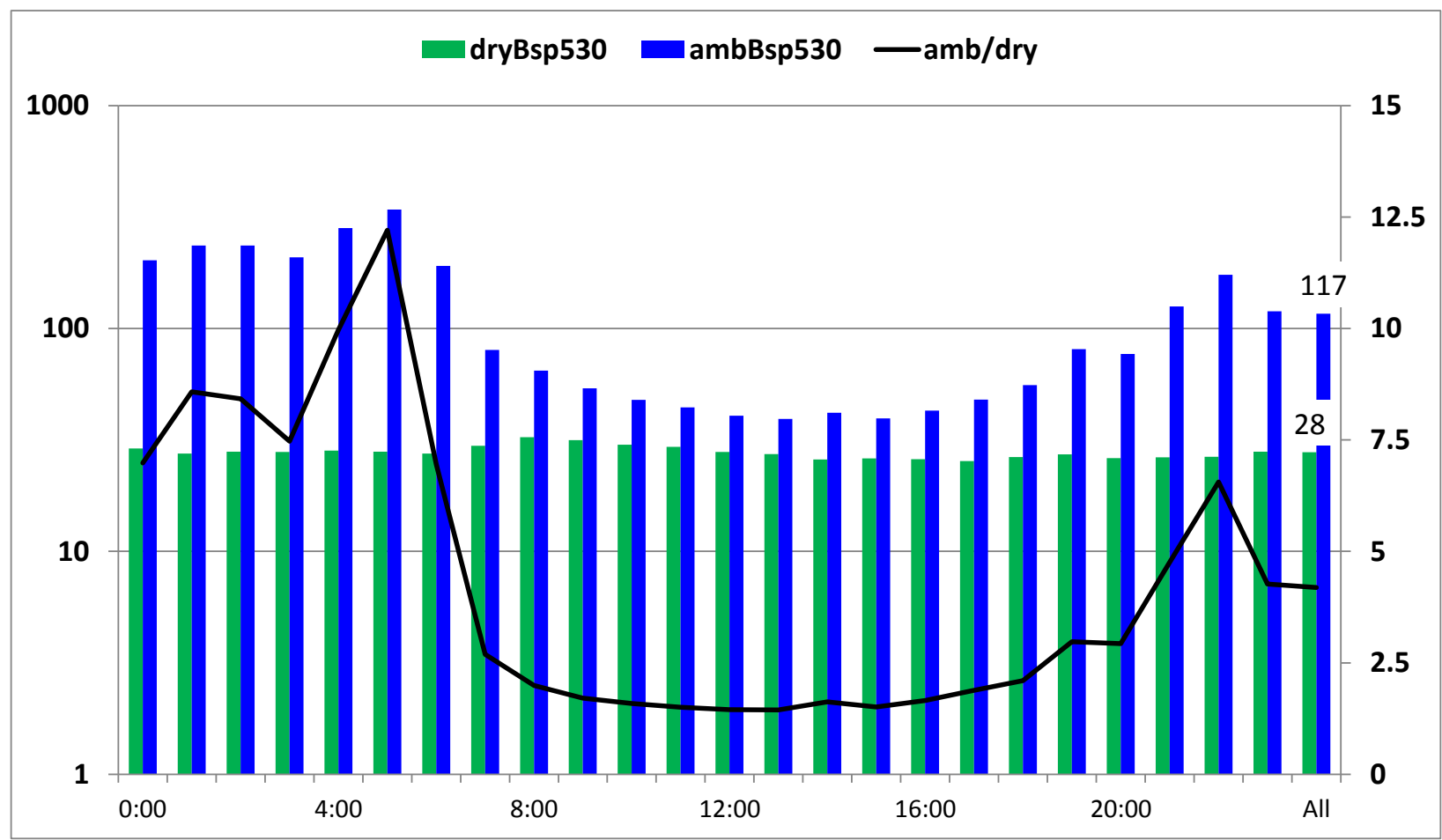

Figure S17. Mean hourly light scattering coefficient $\left(b_{\mathrm{sp}}\right)$ for ambient and dried aerosol (left axis, $\mathrm{Mm}^{-1}$ ) versus hour local time during June 1 - July 15, 2013. Ambient and dry period means $\left(117,28 \mathrm{Mm}^{-1}\right.$, respectively) are shown as rightmost bar. The ratio of ambient to dry light scattering (line, right axis) indicates response, with equilibration, of particles at CTR to RH. 

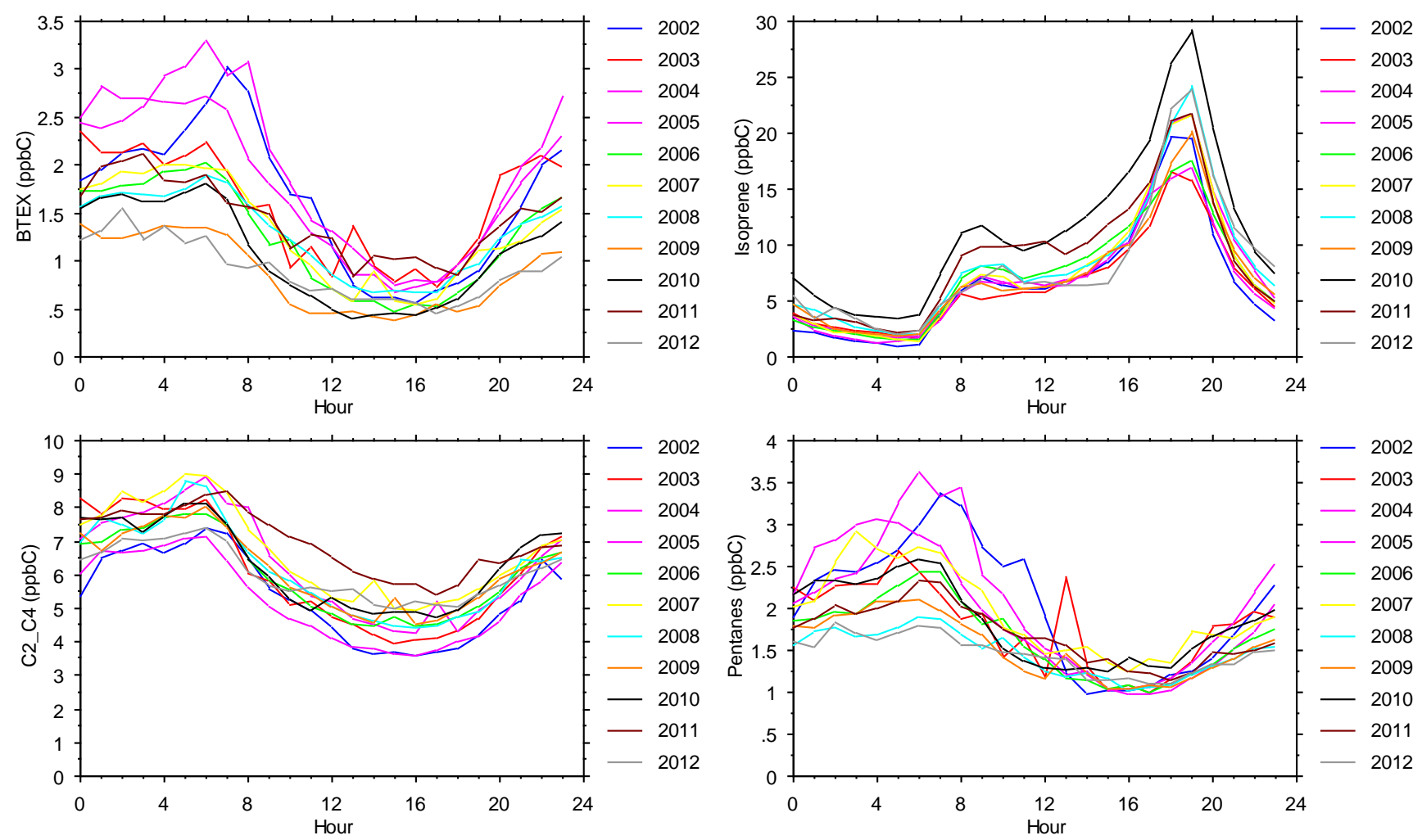

Figure S18. Mean diurnal LST concentrations of BTEX compounds, isoprene, $\mathrm{C}_{2}-\mathrm{C}_{4}$ alkanes, and pentanes, at YRK, showing differences between variations of biogenic isoprene anthropogenic species concentrations. Measurements were made by the PAMS network from June 1 through August 31 of each year. The NMOC concentrations show strong differences between the anthropogenic species and naturally occurring isoprene (Data described in EPA, 1999). 


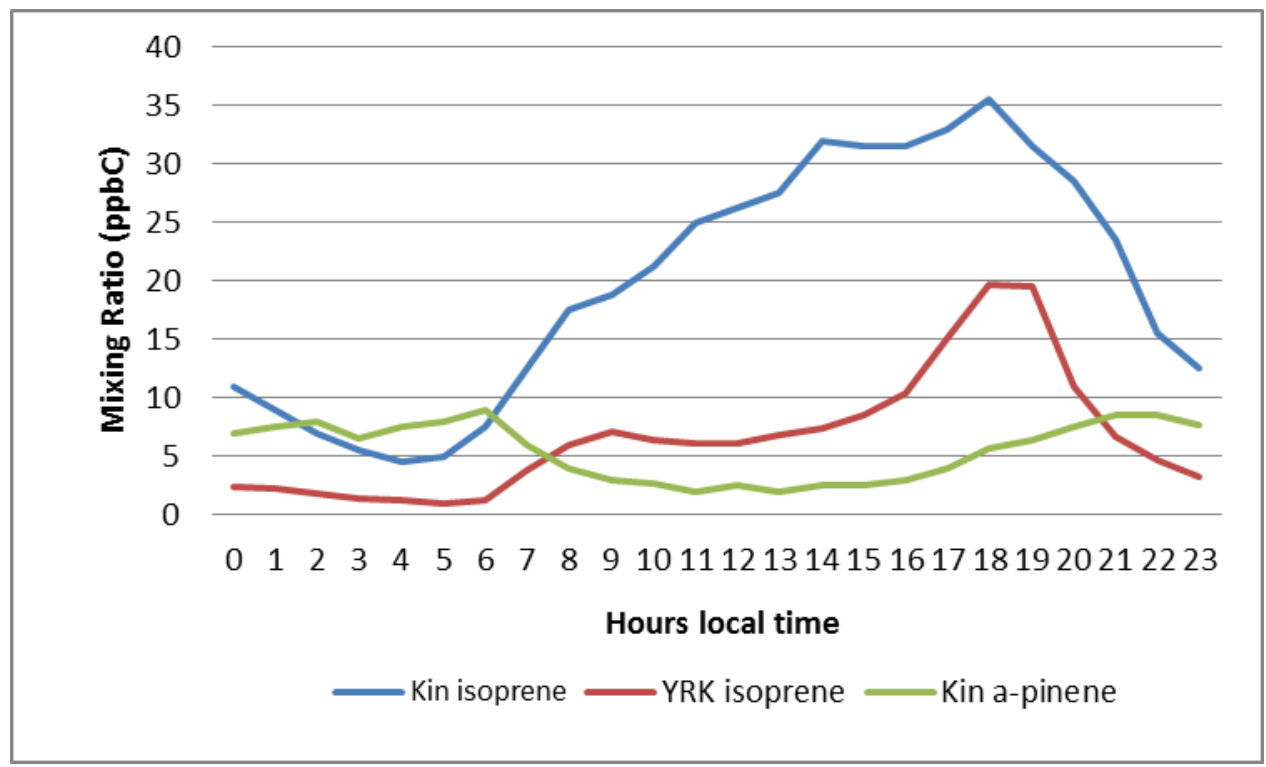

Figure S19. Average isoprene and $\alpha$-pinene concentrations in ppbC from rural Kinterbish State Park, (western) Alabama taken in June-July 1990 (Goldan et al., 1995) compared with YRK PAMS data in summer 2002. The diurnal (LST) variation in isoprene indicates an early morning increase in concentration with increased emissions and mixing with inversion breakup, followed by dilution and photochemical oxidation at midday, ending with a maximum before decline to nighttime levels. This occurs in both the Kinterbish data and at YRK; the YRK late afternoon maximum is lower than shown at Kinterbish. The YRK pattern also is seen at the other PAMS sites (Conyers and S. DeKalb) around Atlanta. The daytime $\alpha$-pinene concentrations (ppbv) are found to be low relative to isoprene, with a morning maximum, mixing during inversion break up, and increased emissions with temperature rise. The $\alpha$-pinene concentrations then decrease through the midday, later rising to a maximum in early evening. 